\title{
On Periodic Solutions of the Incompressible Navier-Stokes Equations on Non-compact Riemannian Manifolds
}

\author{
Thieu Huy Nguyen*, Truong Xuan Pham, Thi Van Nguyen and Thi Ngoc Ha Vu
}

\begin{abstract}
In this paper, we study the existence, uniqueness and stability of the time periodic mild solutions to the incompressible Navier-Stokes equations on the noncompact manifolds with negative Ricci curvature tensor. In our strategy, we combine the dispersive and smoothing estimates for Stokes semigroups and Massera-type theorem to establish the existence and uniqueness of the time periodic mild solution to Stokes equation on Riemannian manifolds. Then using fixed point arguments, we can pass to semilinear equations to obtain the existence and uniqueness of the periodic solution to the imcompressible Navier-Stokes equations under the action of a periodic external force. The stability of the solution is also proved by using the cone inequality.
\end{abstract}

\section{Introduction and statements of main results}

\subsection{Introduction}

The problem of time-periodicity of solutions to Navier-Stokes equations has its long histories. Early results can be traced back to Serrin [31] who proved that the exponential stability of solutions to Navier-Stokes equations (NSE) implies the existence of timeperiodic solutions to NSE in bounded domains. The method of Serrin served as a starting point for many researches on periodic solutions to NSE. This direction has been extended further by Miyakawa and Teramoto [25, Kaniel and Shinbrot [17, and references therein. Then, Maremonti has proved in 23 the stability and existence of periodic solutions to NSE on the whole space. Kozono and Nakao 20 have introduced a new notion of mild solutions and proved the existence of such a solution to NSE on the whole time-line $\mathbb{R}$ in $\mathbb{R}^{d}$ for $d \geq 4$. Taniuchi 32 has the proved the asymptotic stability of such periodic solutions.

Other techniques known formally as "invading domains" have been introduced by Prodi [29], Prouse [30], Yudovich [37, and Heywood [15] used to prove the existence of periodic solutions to NSE on certain unbounded domains. The existence results of such solutions to

Received March 11, 2021; Accepted December 15, 2021.

Communicated by Jenn-Nan Wang.

2020 Mathematics Subject Classification. Primary: 35Q30, 35B10; Secondary: 76D07.

Key words and phrases. Navier-Stokes equations, non-compact Riemannian manifolds, negative Ricci curvature tensors, periodic solutions, stability.

*Corresponding author. 
NSE were shown by Maremonti and Padula 24 on certain exterior domain with symmetric property and small complement. Then, extending further Serrin's method, Galdi and Sohr [10] have proved the existence of periodic solutions to NSE in any exterior domain using the spaces featuring the decay of the solutions at spatial infinity. Yamazaki [36] used interpolation spaces and the iteration scheme method [12,18, to show the existence and uniqueness of periodic mild solutions on exterior domains. For further results in exterior domains, we refer to Taniuchi 33 and van Baalen and Wittwer 35], Galdi and Silvestre [9]. Moreover, Nguyen [26] used the mean-ergodic methods and Massera's principle to show the existence and polynomial stability of periodic solutions to the NSE around a rotating obstacle. For recent results concerning periodic solutions to NSE in the whole space or past moving cylinders, we refer the reader to Galdi [7,8]. A general approach to the problem of periodic solutions to fluid flow problems using interpolation spaces and smoothness of corresponding linearized equations has been introduced by Geissert, Hieber and Nguyen [11.

In the present paper we will study the existence and stability of periodic solutions to incompressible Navier-Stokes equations (NSE) for vector fields in a non-compact Riemannian manifold $(\mathcal{M}, g)$ with negative Ricci curvature tensor. Concretely, denoting $\Gamma(T \mathcal{M})$ the set of all vector fields on $\mathcal{M}$, we consider NSE for a vector-field-valued mapping $u: \mathbb{R}_{+} \times \mathcal{M} \rightarrow \Gamma(T \mathcal{M})$

$$
\left\{\begin{array}{l}
\partial_{t} u+\nabla_{u} u+\nabla \pi=\vec{\Delta} u+r(u)+f \\
\operatorname{div} u=0 \\
u(0, x)=u_{0}(x) \in \Gamma(T \mathcal{M}) \quad \text { for all } x \in \mathcal{M}
\end{array}\right.
$$

where $\nabla$ denotes the Levi-Civita connection on $\mathcal{M} ; \pi$ is the pressure; $r(u)$ is Ricci operator (see the definition in the next section); $f$ is external force; and $\vec{\Delta}$ is Bochner Laplace operator. We refer the reader to $[1,2,19,21,38$ for the well-posedness and ill-posedness results for NSE on non-compact Riemannian manifolds. For the case of Einstein manifolds with negative curvature tensor, in [27] we obtained the existence and stability of periodic solutions to Navier-Stokes equations. In the present paper we will extent such results to the case of general noncompact Riemannian manifolds satisfying the hypotheses (H1)-(H4) below.

We would like to note that on a non-compact Riemannian manifold with negative curvature tensor, the study of the Stokes problem may be transformed to the case of the vectorial heat equations. Based on this important fact, Pierfelice [28] has proved the dispersive and smoothing estimates for the vectorial heat and Stokes semigroup associated with the Bochner Laplacian on non-compact Riemannian manifolds with negative curvature. Actually, Pierfelice 28] obtained exponential decaying and $L^{p}-L^{2}$ estimates for 
Stokes semigroups on Riemannian manifolds with negative curvatures. Using these estimates and Kato-iteration methods, Pierfelice obtained the well-posedness of Navier-Stokes equations for vector fields on non-compact Riemannian manifolds (see details in [28]). However, since the energy inequalities are not valid for NSE on general non-compact Riemannian manifolds (see [2, Theorems 3.2, 3.3]), the stability estimates for semilinear equations using energy inequalities cannot directly be applied. Moreover, we are working here with $L^{p}$-phase space with general $p$, then the usual estimates in $L^{2}$ spaces using scalar product cannot be used. Therefore, in the present paper, we use dispersive and smoothing estimates of the corresponding linearized Stokes equation in combination with Masseratype theorem to obtain the existence and uniqueness of a periodic solution to linearized Stokes equation, and then we pass to the case of semilinear Navier-Stokes equations using fixed-point arguments. Using cone inequality we prove the exponential stability of the periodic solution to NSE.

\subsection{Statements of the main results}

Using the Kodaira-Hodge operator $\mathbb{P}=I+\operatorname{grad}\left(-\Delta_{g}\right)^{-1}$ div we can get rid of the pressure term $\pi$ and then obtain from (1.1) that

$$
\left\{\begin{array}{l}
\partial_{t} u-(\vec{\Delta} u+r(u)+G(u))=\mathbb{P}\left[-\nabla_{u} u+f\right], \\
\operatorname{div} u=0, \\
u(0, x)=u_{0}(x) \in \Gamma(T M) \text { for all } x \in \mathcal{M} ; \operatorname{div} u_{0}=0,
\end{array}\right.
$$

where $G(u)=2 \operatorname{grad}\left(-\Delta_{g}\right)^{-1} \operatorname{div}(r(u))$ with $\Delta_{g}$ being the Laplace-Beltrami operator. The corresponding inhomogeneous Stokes equation takes the form

$$
\left\{\begin{array}{l}
\partial_{t} u=-\mathcal{A} u+\mathbb{P}\left[-\nabla_{v} v+f\right] \\
u(0, x)=u_{0}(x) \in \Gamma(T \mathcal{M}) \quad \text { for all } x \in \mathcal{M}
\end{array}\right.
$$

for given vector-field valued mappings $v(t, \cdot)$ and $f(t, \cdot) \in \Gamma(T \mathcal{M})$, where $\mathcal{A} u=-(\vec{\Delta} u+$ $r(u)+G(u))$, and $e^{-t \mathcal{A}}$ is denoted the semigroup associated with the homogeneous Cauchy problem

$$
\left\{\begin{array}{l}
\partial_{t} u=-\mathcal{A} u \\
u(0, x)=u_{0}(x) \in \Gamma(T \mathcal{M}) \quad \text { for all } x \in \mathcal{M}
\end{array}\right.
$$

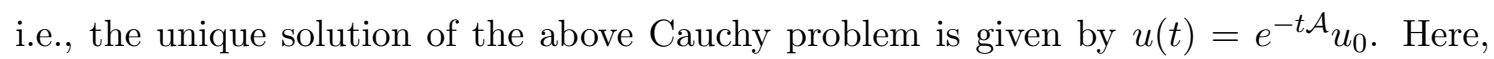
traditionally, we denote $u(t)$ for $u(t, \cdot)$. To state our main results we need the following notion of mild solution. 
Definition 1.1. By a mild solution to 1.3 we mean a mapping $u: \mathbb{R}_{+} \times \mathcal{M} \rightarrow \Gamma(T \mathcal{M})$ which satisfies the integral equation

$$
u(t)=e^{-t \mathcal{A}} u_{0}+\int_{0}^{t} e^{-(t-\tau) \mathcal{A}} \mathbb{P}\left[-\nabla_{v} v+f\right](\tau) d \tau \quad \text { for } t \geq 0 .
$$

Moreover, for a given Banach space $X$ we denote the following Banach space

$$
C_{b}\left(\mathbb{R}_{+}, X\right):=\left\{h: \mathbb{R}_{+} \rightarrow X \mid h \text { is continuous and } \sup _{t \in \mathbb{R}_{+}}\|h(t)\|_{X}<\infty\right\}
$$

endowed with the norm $\|h\|_{\infty, X}=\|h\|_{C_{b}\left(\mathbb{R}_{+}, X\right)}:=\sup _{t \in \mathbb{R}_{+}}\|h(t)\|_{X}$.

We consider the existence and stability of the periodic mild solutions of equations 1.2 , (1.3) on the following Banach space

$$
\begin{aligned}
\mathcal{X}:= & \left\{u \in C_{b}\left(\mathbb{R}_{+}, L^{p}(\Gamma(T \mathcal{M})) \cap L^{2}(\Gamma(T \mathcal{M}))\right), \nabla u \in C_{b}\left(\mathbb{R}_{+}, L^{\widetilde{p}}(\Gamma(T \mathcal{M})) \cap L^{s}(\Gamma(T \mathcal{M}))\right) \mid\right. \\
& \text { the function } t \mapsto\|u(t)\|_{2}+\|u(t)\|_{L^{p}}+\left[c_{d}(t)\right]^{-\left(\frac{1}{p}-\frac{1}{\widetilde{p}}+\frac{1}{d}\right)}\|\nabla u(t)\|_{L^{\widetilde{p}}} \\
& \left.+\left[c_{d}(t)\right]^{-\left(\frac{1}{p}-\frac{1}{s}+\frac{1}{d}\right)}\|\nabla u(t)\|_{L^{s}} \text { belongs to } L^{\infty}\left(\mathbb{R}_{+}\right)\right\}
\end{aligned}
$$

endowed with the norm

$$
\begin{aligned}
&\|u\|_{\mathcal{X}}:=\sup _{t \in \mathbb{R}_{+}}(\|u(t)\|_{L^{2}}+\|u(t)\|_{L^{p}}+\left[c_{d}(t)\right]^{-\left(\frac{1}{p}-\frac{1}{\widetilde{p}}+\frac{1}{d}\right)}\|\nabla u(t)\|_{L^{\widetilde{p}}} \\
&\left.+\left[c_{d}(t)\right]^{-\left(\frac{1}{p}-\frac{1}{s}+\frac{1}{d}\right)}\|\nabla u(t)\|_{L^{s}}\right) .
\end{aligned}
$$

Here we assume that $d<p<\widetilde{p}<s$ where $\frac{1}{2}=\frac{1}{p}+\frac{1}{\widetilde{p}}$, and denote $\|\cdot\|_{L^{p}}:=\|\cdot\|_{L^{p}(\Gamma(T \mathcal{M}))}$, whereas $c_{d}(t):=C_{0} \max \left\{\frac{1}{t^{\frac{d}{2}}}, 1\right\}$.

We now state our first main result on the existence of the periodic mild solution to (1.3) in the following theorem.

Theorem 1.2. Let $(\mathcal{M}, g)$ be a d-dimensional non-compact manifold with negative Ricci curvature tensor. Suppose that $v \in \mathcal{X}$ and the external force $f \in C_{b}\left(\mathbb{R}_{+}, L^{p}(\Gamma(T \mathcal{M})) \cap\right.$ $\left.L^{2}(\Gamma(T \mathcal{M}))\right)(p>d)$ are $T$-periodic functions. Then, problem (1.3) has one and only one $T$-periodic mild solution $\widehat{u} \in \mathcal{X}$ satisfying

$$
\|\widehat{u}\|_{\mathcal{X}} \leq(\widetilde{C}+1)\left(\widetilde{C}\|f\|_{L^{p} \cap L^{2}}+\widetilde{M}\|v\|_{\mathcal{X}}^{2}\right)
$$

Analogously to the case of linearized Stokes equations, by a mild solution to equation (1.2) we mean the vector-field-valued map $u: \mathbb{R}_{+} \times \mathcal{M} \rightarrow \Gamma(T \mathcal{M})$ satisfying the integral equation

$$
u(t)=e^{-t \mathcal{A}} u_{0}+\int_{0}^{t} e^{-(t-\tau) \mathcal{A}} \mathbb{P}\left[\left(-\nabla_{u} u+f\right)(\tau)\right] d \tau \quad \text { for } t \geq 0 .
$$

We then state our second main result on the existence and uniqueness of the periodic mild solution to 1.2 in the following theorem. 
Theorem 1.3. Let $(\mathcal{M}, g)$ be a d-dimensional non-compact manifold with the negative Ricci curvature tensor and let $f \in C_{b}\left(\mathbb{R}_{+}, L^{p}(\Gamma(T \mathcal{M})) \cap L^{2}(\Gamma(T \mathcal{M}))\right)(p>d)$ be $T$ periodic with respect to $t$. Then, if $\|f\|_{L^{p} \cap L^{2}}$ is sufficiently small, the equation (1.2) has one and only one T-periodic mild solution $\widehat{u}$ on a small ball of $\mathcal{X}$.

Lastly, our third main result on the exponential stability of the periodic mild solution is stated as follows.

Theorem 1.4. The T-periodic mild solution $\widehat{u}$ of equation $(1.2)$ is exponentially stable in the sense that for any other mild solution $u \in \mathcal{X}$ to 11.2 such that $\|u(0)-\widehat{u}(0)\|_{L^{p} \cap L^{2}}$ is small enough, we have

$$
\||| u(t)-\widehat{u}(t) \mid\| \leq C_{\gamma} e^{-\gamma t}\|u(0)-\widehat{u}(0)\|_{L^{p} \cap L^{2}} \quad \text { for all } t>0,
$$

here we denote

$$
\begin{aligned}
\|\| u(t)\|\|:= & \|u(t)\|_{2}+\|u(t)\|_{L^{p}}+\left[c_{d}(t)\right]^{-\left(\frac{1}{p}-\frac{1}{\tilde{p}}+\frac{1}{d}\right)}\|\nabla u(t)\|_{L^{\widetilde{p}}} \\
& +\left[c_{d}(t)\right]^{-\left(\frac{1}{p}-\frac{1}{s}+\frac{1}{d}\right)}\|\nabla u(t)\|_{L^{s}}
\end{aligned}
$$

whereas $\gamma$ is a positive constant satisfying $0<\gamma<\beta$, and $C_{\gamma}$ is a constant independent of $u$ and $\widehat{u}$.

We will give the proofs of our main results in Section 3 .

2. Intermezzo on the incompressible Navier-Stokes equations on non-compact Riemannian manifolds

For $d \in \mathbb{N}, d \geq 2$, we consider a $d$-dimensional non-compact Riemannian manifold $(\mathcal{M}, g)$ whose the Ricci tensor Ric is a negative. We restrict ourselves to the case that the sectional curvature of $(\mathcal{M}, g)$ is negative, so that the smoothing and dispersive estimates obtained by Pierfelice [28] can be applied. We refer the reader to [13, 16 for notions and detail discussions on Riemannian manifolds and related concepts of geometric analysis. For details on Navier-Stokes equations on Riemannian manifolds we refer to [6, 28, 34 and references therein. For the reader's convenience, in what follows, we recall some notions on differential operators on Riemannian manifolds. We denote the Levi-Civita connection by $\nabla$ and the set of all vector fields on $\mathcal{M}$ by $\Gamma(T \mathcal{M})$. For $X \in \Gamma(T \mathcal{M})$ we can extend $\nabla_{X}$ to arbitrary $(p, q)$ tensor by requiring

(i) $\nabla_{X}(c(S))=c\left(\nabla_{X} S\right)$ for any contraction $c$,

(ii) $\nabla_{X}(S \otimes T)=\nabla_{X} S \otimes T+S \otimes \nabla_{X} T$, 
where we take the convention that $\nabla_{X} f=X \cdot f$ for a function $f: \mathcal{M} \rightarrow \mathbb{R}$.

In particular, for $S \in \Gamma\left(\otimes^{p}(T \mathcal{M}) \otimes^{q}\left(T^{*} \mathcal{M}\right)\right)$ we get

$$
\begin{aligned}
\left(\nabla_{X} S\right)\left(X_{1}, \ldots, X_{q}\right)= & \nabla_{X}\left(S\left(X_{1}, \ldots, X_{q}\right)\right)-S\left(\nabla_{X} X_{1}, \ldots, X_{q}\right) \\
& -\cdots-S\left(X_{1}, \ldots, \nabla_{X} X_{q}\right)
\end{aligned}
$$

Moreover, we define the covariant derivatives $\nabla$ on tensor field $S \in \Gamma\left(\otimes^{p}(T \mathcal{M}) \otimes^{q}\left(T^{*} \mathcal{M}\right)\right)$ by

$$
\nabla S\left(X, X_{1}, \ldots, X_{q}\right)=\left(\nabla_{X} S\right)\left(X_{1}, \ldots, X_{q}\right),
$$

hence $\nabla S \in \Gamma\left(\otimes^{p}(T \mathcal{M}) \otimes^{q+1}\left(T^{*} \mathcal{M}\right)\right)$. Next, we recall the "music notations" on Riemannian manifolds. For a 1 -form $w$, we define the vector field $w^{\sharp}$ by

$$
g\left(w^{\sharp}, Y\right)=w(Y), \quad \forall Y \in \Gamma(T \mathcal{M})
$$

whereas, for a vector field $X$, we define the 1 -form $X^{b}$ by

$$
X^{b}(Y)=g(X, Y), \quad \forall Y \in \Gamma(T \mathcal{M}) .
$$

The metric on 1 -forms can then be defined by setting $g(w, \eta):=g\left(w^{\sharp}, \eta^{\sharp}\right), \forall w, \eta \in$ $\Gamma\left(T^{*} \mathcal{M}\right)$. The Riemannian gradient of a function is then defined as

$$
\operatorname{grad} p=(d p)^{\sharp} \text {. }
$$

More generally, for $(p, q)$-tensor field $T \in \Gamma\left(\otimes^{p}(T \mathcal{M}) \otimes^{q}\left(T^{*} \mathcal{M}\right)\right)$ we have

$$
\begin{aligned}
T^{\sharp} & =C_{1}^{2}\left(g^{-1} \otimes T\right) \in \Gamma\left(\otimes^{p+1}(T \mathcal{M}) \otimes^{q-1}\left(T^{*} \mathcal{M}\right)\right), \\
T^{b} & =C_{2}^{1}(g \otimes T) \in \Gamma\left(\otimes^{p-1}(T \mathcal{M}) \otimes^{q+1}\left(T^{*} \mathcal{M}\right)\right), \\
\operatorname{div} T & =C_{1}^{1} \nabla T \in \Gamma\left(\otimes^{p-1}(T \mathcal{M}) \otimes^{q}\left(T^{*} \mathcal{M}\right)\right),
\end{aligned}
$$

where $C_{j}^{i}$ stands for the contraction of the $i$ and $j$ indices for tensors.

Next, the Laplace-Beltrami operator $\Delta_{g}$ applying on functions is defined as

$$
\Delta_{g}(f)=\operatorname{div} \operatorname{grad} f=\frac{1}{\sqrt{|g|}} \frac{\partial}{\partial x^{j}}\left(\sqrt{|g|} g^{i j} \frac{\partial f}{\partial x^{i}}\right)
$$

for a function $f: \mathcal{M} \rightarrow \mathbb{R}$, where $|g|=\operatorname{det} g$.

Furthermore, the vectorial Laplacian $L$ is defined by the stress tensor (see [6, 34]):

$$
L u=\operatorname{div}\left(\nabla u+\nabla u^{t}\right)^{\sharp} .
$$

Since $\operatorname{div} u=0$ we can express $L$ in the following formula

$$
L u=\vec{\Delta} u+r(u)
$$


where $\vec{\Delta}$ is the Bochner-Laplacian

$$
\vec{\Delta} u=-\nabla^{*} \nabla u=\operatorname{Tr}_{g}\left(\nabla^{2} u\right)
$$

and $r(\cdot)$ is the Ricci operator related to the Ricci curvature tensor by the formula

$$
r(u)=(\operatorname{Ric}(u, \cdot))^{\sharp} \quad \text { for all } u \in \Gamma(T \mathcal{M}),
$$

where the Ricci curvature tensor is defined by

$$
\operatorname{Ric}(X, Y)=\sum_{i=1}^{d} g\left(\mathcal{R}\left(X, e_{i}\right) Y, e_{i}\right) \quad \text { for all } X, Y \in \Gamma(T \mathcal{M})
$$

for the standard basis $\left\{e_{i}=\frac{\partial}{\partial x_{i}}\right\}_{i=1}^{d}$ and $\mathcal{R}$ being the curvature tensor on $M$ defined by

$$
\mathcal{R}(X, Y) Z:=-\nabla_{X}\left(\nabla_{Y} Z\right)+\nabla_{Y}\left(\nabla_{X} Z\right)+\nabla_{[X, Y]} Z \text { for all } X, Y, Z \in \Gamma(T \mathcal{M})
$$

Moreover, the sectional curvature $\kappa$ is defined as $\kappa(X, Y):=\frac{\mathcal{R}(X, Y, X, Y)}{g(X, X) g(Y, Y)-g(X, Y)^{2}}$ for all $X, Y \in T_{x} \mathcal{M}$.

Now, for a smooth, complete, non-compact, simply connected Riemannian manifold $(\mathcal{M}, g)$ we state the following hypotheses which were introduced in 28:

$\left(\mathrm{H}_{1}\right)|\mathcal{R}|+|\nabla \mathcal{R}|+\left|\nabla^{2} \mathcal{R}\right| \leq K$

$\left(\mathrm{H}_{2}\right)-\frac{1}{c_{0}} g \leq \mathrm{Ric} \leq-c_{0} g$ for some $c_{0}$ positive,

$\left(\mathrm{H}_{3}\right) \kappa<0$,

$\left(\mathrm{H}_{4}\right) \inf _{x \in \mathcal{M}} r_{x}>0$

where $r_{x}$ is the injectivity radius for the exponential map at $x$.

There are several Riemannian manifolds satisfying the above hypotheses $\left(\mathrm{H}_{1}\right)-\left(\mathrm{H}_{4}\right)$ such as real hyperbolic manifolds, non-compact Einstein manifolds with negative Ricci curvature tensors (see 14, 16]), Damek-Ricci manifolds (see [4]) and symmetric manifolds of non-compact types (see [5, 14]).

In this paper, we study the periodic solutions to the Cauchy problem for the incompressible Navier-Stokes equations (1.1) on $\mathcal{M}$.

Remark 2.1. We notice that the operator $\vec{\Delta}+r+G$ does not commute with the KodairaHodge operator $\mathbb{P}=I+\operatorname{grad}\left(-\Delta_{g}\right)^{-1}$ div on the generalized non-compact manifolds with all the conditions $\left(\mathrm{H}_{1}\right)-\left(\mathrm{H}_{4}\right)$. 


\section{Proofs of main results}

In this section, we will give the proofs of our three main results stated in the first section. We first prove the existence of bounded mild solutions to linear inhomogeneous Stokes equations 1.3 with bounded external forces. To that purpose we need the $L^{p}-L^{q}$ dispersive and smoothing estimates of the semigroup $e^{-t \mathcal{A}}$. These estimates have been proved for semigroups on noncompact manifolds satisfying $\left(\mathrm{H}_{1}\right)-\left(\mathrm{H}_{4}\right)$ by Pierfelice in [28]. We now recall the results on the dispersive and smoothing estimates obtained in 28 for the semigroup $e^{-t \mathcal{A}}$ in the following lemma.

Lemma 3.1. [28, Corollary 4.13 and Theorem 4.15] Assuming $\left(\mathrm{H}_{1}\right)-\left(\mathrm{H}_{4}\right)$, putting $c_{d}(t)=$ $C_{0} \max \left(\frac{1}{t^{d / 2}}, 1\right)$. There exist $\beta \geq C_{0}>0$ and some $C>0$ such that the solution of (1.4) satisfies the following dispersive and smoothing estimates:

(i) For $2 \leq p \leq r<+\infty$ and for all $u_{0} \in L^{p}(\Gamma(T \mathcal{M})) \cap L^{2}(\Gamma(T \mathcal{M}))$,

$$
\begin{gathered}
\left\|e^{-t \mathcal{A}} u(t)\right\|_{L^{r}} \leq C\left[c_{d}(t)\right]^{\frac{1}{p}-\frac{1}{r}} e^{-\beta t}\left(\left\|u_{0}\right\|_{L^{p}}+\left\|u_{0}\right\|_{L^{2}}\right), \quad \forall t>0, \\
\left\|\nabla e^{-t \mathcal{A}} u(t)\right\|_{L^{r}} \leq C\left[c_{d}(t)\right]^{\frac{1}{p}-\frac{1}{r}+\frac{1}{d}} e^{-\beta t}\left(\left\|u_{0}\right\|_{L^{p}}+\left\|u_{0}\right\|_{L^{2}}\right), \quad \forall t>0 .
\end{gathered}
$$

(ii) For $1<p \leq 2 \leq r<+\infty$ and for all $u_{0} \in L^{p}(\Gamma(T \mathcal{M}))$,

$$
\begin{gathered}
\left\|e^{-t \mathcal{A}} u(t)\right\|_{L^{r}} \leq C\left[c_{d}(t)\right]^{\frac{1}{p}-\frac{1}{r}} e^{-\beta t}\left\|u_{0}\right\|_{L^{p}}, \quad \forall t>0, \\
\left\|\nabla e^{-t \mathcal{A}} u(t)\right\|_{L^{r}} \leq C\left[c_{d}(t)\right]^{\frac{1}{p}-\frac{1}{r}+\frac{1}{d}} e^{-\beta t}\left\|u_{0}\right\|_{L^{p}}, \quad \forall t>0 .
\end{gathered}
$$

The following lemma gives us the boundedness (in time) of mild solutions to 1.3 for each bounded external force.

Lemma 3.2. Consider Stokes equation (1.3) on d-dimensional non-compact manifold $(\mathcal{M}, g)$ with negative Ricci curvature tensor. Let $u_{0} \in L^{p}(\Gamma(T \mathcal{M})) \cap L^{2}(\Gamma(T \mathcal{M}))(p>d)$ and suppose that $v \in \mathcal{X}, f \in C_{b}\left(\mathbb{R}_{+}, L^{p}(\Gamma(T \mathcal{M})) \cap L^{2}(\Gamma(T \mathcal{M}))\right)$. Then, the problem (1.3) has one and only one mild solution $u \in \mathcal{X}$ given by the formula 1.5 with $u(0)=u_{0}$. Furthermore, we have

$$
\|u\|_{\mathcal{X}} \leq \widetilde{C}\left(\left\|u_{0}\right\|_{L^{p} \cap L^{2}}+\|f\|_{\infty, L^{p} \cap L^{2}}\right)+\widetilde{M}\|v\|_{\mathcal{X}}^{2}
$$

for some constants $\widetilde{C}$ and $\widetilde{M}$ independent of $u_{0}, u, v$ and $f$. Here we denote the norm $\|\cdot\|_{L^{p}(\Gamma(T \mathcal{M})) \cap L^{2}(\Gamma(T \mathcal{M}))}$ by $\|\cdot\|_{L^{p} \cap L^{2}}$ and the norm $\|\cdot\|_{C_{b}\left(\mathbb{R}_{+}, L^{p}(\Gamma(T \mathcal{M})) \cap L^{2}(\Gamma(T \mathcal{M}))\right)}$ by $\|\cdot\|_{\infty, L^{p} \cap L^{2}}$.

Proof. Consider the function $u$ defined by the formula 1.5 with $u(0)=u_{0}$. We now estimate the norm $\|u\|_{\mathcal{X}}$. For simplicity of writing, we denote $\|\cdot\|_{p}:=\|\cdot\|_{L^{p}(\Gamma(T \mathcal{M}))}$. 
On Riemannian non-compact manifold with negative curvature tensor we have the $L^{p}$ boundedness of Riesz transform (see [22]), therefore the operator $\mathbb{P}$ is bounded. Using the first inequality in assertion (i) of Lemma 3.1 we have

$$
\begin{aligned}
\|u(t)\|_{2} & \leq\left\|e^{-t \mathcal{A}} u_{0}\right\|_{2}+\int_{0}^{t}\left\|e^{-(t-\tau) \mathcal{A}} \mathbb{P}\left[\nabla_{v} v+f\right](\tau)\right\|_{2} d \tau \\
& \leq C e^{-\beta t}\left\|u_{0}\right\|_{2}+\int_{0}^{t} e^{-\beta(t-\tau)}\left\|\nabla_{v} v(\tau)+f(\tau)\right\|_{2} d \tau \\
& \leq C\left\|u_{0}\right\|_{L^{p} \cap L^{2}}+\left(\int_{0}^{t} e^{-\beta(t-\tau)}\left(\|v(\tau)\|_{p}\|\nabla v(\tau)\|_{\widetilde{p}}+\|f\|_{2}\right) d \tau\right) \\
& \leq C\left\|u_{0}\right\|_{L^{p} \cap L^{2}}+\left(\int_{0}^{t} e^{-\beta(t-\tau)} d \tau\|f\|_{\infty, L^{2}}+\int_{0}^{t} e^{-\beta(t-\tau)}\left[c_{d}(\tau)\right]^{\frac{1}{p}-\frac{1}{\tilde{p}}+\frac{1}{d}} d \tau\|v\|_{\mathcal{X}}^{2}\right) \\
& \leq C\left\|u_{0}\right\|_{L^{p} \cap L^{2}}+\frac{N}{\beta}\left(1-e^{-\beta t}\right)\|f\|_{\infty, L^{2}}+G_{1}(t)\|v\|_{\mathcal{X}}^{2} \\
& \leq C_{1}\left(\left\|u_{0}\right\|_{L^{p} \cap L^{2}}+\|f\|_{\infty, L^{p} \cap L^{2}}\right)+M_{1}\|v\|_{\mathcal{X}}^{2},
\end{aligned}
$$

where $C_{1}:=\max \left\{C, \frac{N}{\beta}\right\}$ and

$$
G_{1}(t)=\int_{0}^{t} e^{-\beta(t-\tau)}\left[c_{d}(\tau)\right]^{\frac{1}{p}-\frac{1}{\tilde{p}}+\frac{1}{d}} \leq M_{1}<+\infty .
$$

We can estimate $G_{1}(t)$ and determine $M_{1}$ as follows:

$$
\begin{aligned}
G_{1}(t) & :=\int_{0}^{t}\left[c_{d}(\tau)\right]^{\frac{1}{p}-\frac{1}{\widetilde{p}}+\frac{1}{d}} e^{-\beta(t-\tau)} d \tau=\int_{0}^{t}\left[\max \left\{\tau^{-\frac{d}{2}}, 1\right\}\right]^{\frac{1}{p}-\frac{1}{\widetilde{p}}+\frac{1}{d}} e^{-\beta(t-\tau)} d \tau \\
& =\left\{\begin{array}{lc}
\int_{0}^{1} \tau^{-\frac{d}{2}\left(\frac{1}{p}-\frac{1}{\widetilde{p}}+\frac{1}{d}\right)} e^{-\beta(t-\tau)} d \tau+\int_{1}^{t} e^{-\beta(t-\tau)} d \tau & \text { if } t \geq 1, \\
\int_{0}^{1} \tau^{-\frac{d}{2}\left(\frac{1}{p}-\frac{1}{\tilde{p}}+\frac{1}{d}\right)} e^{-\beta(t-\tau)} d \tau & \text { if } 0<t<1
\end{array}\right. \\
& \leq \int_{0}^{1} \tau^{-\frac{d}{2}\left(\frac{1}{p}-\frac{1}{\widetilde{p}}+\frac{1}{d}\right)} d \tau+\left(\frac{1}{\beta}-\frac{1}{\beta} e^{\beta(1-t)}\right)=\frac{1}{\frac{1}{2}-\frac{d}{2 p}+\frac{d}{2 \widetilde{p}}}+\left(\frac{1}{\beta}-\frac{1}{\beta} e^{\beta(1-t)}\right) \leq M_{1},
\end{aligned}
$$

where $M_{1}:=\frac{1}{\frac{1}{2}-\frac{d}{2 p}+\frac{d}{2 \widetilde{p}}}+\frac{1}{\beta}$. Note that since $d<p<\widetilde{p}$, it follows that $\frac{1}{2}-\frac{d}{2 p}+\frac{d}{2 \widetilde{p}}>0$.

Putting $\frac{1}{r}=\frac{1}{p}+\frac{1}{s}$ and using again the first inequality in item (i) of Lemma 3.1, we have

$$
\begin{aligned}
\|u(t)\|_{p} \leq & C e^{-\beta t}\left\|u_{0}\right\|_{L^{p} \cap L^{2}}+\int_{0}^{t} N e^{-\beta(t-\tau)}\|f(\tau)\|_{L^{p} \cap L^{2}} d \tau \\
& +\int_{0}^{t}\left[c_{n}(t-\tau)\right]^{\frac{1}{r}-\frac{1}{p}} e^{-\beta(t-\tau)}\left(\left\|\nabla_{v} v(\tau)\right\|_{r}+\left\|\nabla_{v} v(\tau)\right\|_{2}\right) d \tau \\
\leq & C\left\|u_{0}\right\|_{L^{p} \cap L^{2}}+N \int_{0}^{t} e^{-\beta(t-\tau)} d \tau\|f\|_{\infty, L^{p} \cap L^{2}} \\
& +\int_{0}^{t}\left[c_{d}(t-\tau)\right]^{\frac{1}{s}} e^{-\beta(t-\tau)}\left(\|v(\tau)\|_{p}\|\nabla v(\tau)\|_{s}+\|v(\tau)\|_{p}\|\nabla v(\tau)\|_{\widetilde{p}}\right) d \tau
\end{aligned}
$$




$$
\begin{aligned}
\leq & C\left\|u_{0}\right\|_{L^{p} \cap L^{2}}+\frac{N}{\beta}\|f\|_{\infty, L^{p} \cap L^{2}} \\
& +\int_{0}^{t}\left[c_{d}(t-\tau)\right]^{\frac{1}{s}}\left(\left[c_{d}(\tau)\right]^{\frac{1}{p}-\frac{1}{s}+\frac{1}{d}}+\left[c_{d}(\tau)\right]^{\frac{1}{p}-\frac{1}{\widetilde{p}}+\frac{1}{d}}\right) e^{-\beta(t-\tau)} d \tau\|v\|_{\mathcal{X}}^{2} \\
\leq & C\left\|u_{0}\right\|_{L^{p} \cap L^{2}}+\frac{N}{\beta}\|f\|_{\infty, L^{p} \cap L^{2}}+G_{2}(t)\|v\|_{\mathcal{X}}^{2} \\
\leq & C_{1}\left(\left\|u_{0}\right\|_{L^{p} \cap L^{2}}+\|f\|_{\infty, L^{p} \cap L^{2}}\right)+M_{2}\|v\|_{\mathcal{X}}^{2},
\end{aligned}
$$

where $C_{1}:=\max \left\{C, \frac{N}{\beta}\right\}$ and

$$
G_{2}(t)=\int_{0}^{t}\left[c_{d}(t-\tau)\right]^{\frac{1}{s}}\left(\left[c_{d}(\tau)\right]^{\frac{1}{p}-\frac{1}{s}+\frac{1}{d}}+\left[c_{d}(\tau)\right]^{\frac{1}{p}-\frac{1}{\widetilde{p}}+\frac{1}{d}}\right) e^{-\beta(t-\tau)} d \tau \leq M_{2}<+\infty .
$$

We can estimate $G_{2}(t)$ and give a explicit value of $M_{2}$ as follows:

$$
\begin{aligned}
G_{2}(t):= & \int_{0}^{t}\left[c_{d}(t-\tau)\right]^{\frac{1}{s}}\left(\left[c_{d}(\tau)\right]^{\frac{1}{p}-\frac{1}{s}+\frac{1}{d}}+\left[c_{d}(\tau)\right]^{\frac{1}{p}-\frac{1}{\tilde{p}}+\frac{1}{d}}\right) e^{-\beta(t-\tau)} d \tau \\
= & \int_{0}^{t}\left[\max \left\{(t-\tau)^{-\frac{d}{2}}, 1\right\}\right]^{\frac{1}{s}}\left(\left[\max \left\{\tau^{-\frac{d}{2}}, 1\right\}\right]^{\frac{1}{p}-\frac{1}{s}+\frac{1}{d}}+\left[\max \left\{\tau^{-\frac{d}{2}}, 1\right\}\right]^{\frac{1}{p}-\frac{1}{\widetilde{p}}+\frac{1}{d}}\right) \\
& \times e^{-\beta(t-\tau)} d \tau .
\end{aligned}
$$

If $0<t \leq 1$ then

$$
\begin{aligned}
& G_{2}(t) \leq \int_{0}^{t}(t-\tau)^{-\frac{d}{2 s}}\left(\tau^{-\frac{d}{2}\left(\frac{1}{p}-\frac{1}{s}+\frac{1}{d}\right)}+\tau^{-\frac{d}{2}\left(\frac{1}{p}-\frac{1}{\tilde{p}}+\frac{1}{d}\right)}\right) d \tau \\
& =\int_{0}^{t}\left(1-\frac{\tau}{t}\right)^{-\frac{d}{2 s}}\left(\frac{\tau}{t}\right)^{-\frac{d}{2}\left(\frac{1}{p}-\frac{1}{s}+\frac{1}{d}\right)} t^{\frac{1}{2}-\frac{d}{2 p}} d\left(\frac{\tau}{t}\right) \\
& +\int_{0}^{1}\left(1-\frac{\tau}{t}\right)^{-\frac{d}{2 s}}\left(\frac{\tau}{t}\right)^{-\frac{d}{2}\left(\frac{1}{p}-\frac{1}{\widetilde{p}}+\frac{1}{d}\right)} t^{\frac{1}{2}-\frac{d}{2}\left(\frac{1}{p}+\frac{1}{s}-\frac{1}{\widetilde{p}}\right)} d\left(\frac{\tau}{t}\right) \\
& \leq \int_{0}^{1}(1-z)^{-\frac{d}{2 s}} z^{-\frac{d}{2}\left(\frac{1}{p}-\frac{1}{s}+\frac{1}{d}\right)} d z+\int_{0}^{1}(1-z)^{-\frac{d}{2 s}} z^{-\frac{d}{2}\left(\frac{1}{p}-\frac{1}{\vec{p}}+\frac{1}{d}\right)} d z \\
& =\int_{0}^{1 / 2}(1-z)^{-\frac{d}{2 s}} z^{-\frac{d}{2}\left(\frac{1}{p}-\frac{1}{s}+\frac{1}{d}\right)} d z+\int_{1 / 2}^{1}(1-z)^{-\frac{d}{2 s}} z^{-\frac{d}{2}\left(\frac{1}{p}-\frac{1}{s}+\frac{1}{d}\right)} d z \\
& +\int_{0}^{1 / 2}(1-z)^{-\frac{d}{2 s}} z^{-\frac{d}{2}\left(\frac{1}{p}-\frac{1}{\vec{p}}+\frac{1}{d}\right)} d z+\int_{1 / 2}^{1}(1-z)^{-\frac{d}{2 s}} z^{-\frac{d}{2}\left(\frac{1}{p}-\frac{1}{\tilde{p}}+\frac{1}{d}\right)} d z \\
& \leq \int_{0}^{1 / 2} 2^{\frac{d}{2 s}} z^{-\frac{d}{2}\left(\frac{1}{p}-\frac{1}{s}+\frac{1}{d}\right)} d z+\int_{1 / 2}^{1}(1-z)^{-\frac{d}{2 s}} 2^{\frac{d}{2}\left(\frac{1}{p}-\frac{1}{s}+\frac{1}{d}\right)} d z \\
& +\int_{0}^{1 / 2} 2^{\frac{d}{2 s}} z^{-\frac{d}{2}\left(\frac{1}{p}-\frac{1}{\tilde{p}}+\frac{1}{d}\right)} d z+\int_{1 / 2}^{1}(1-z)^{-\frac{d}{2 s}} 2^{\frac{d}{2}\left(\frac{1}{p}-\frac{1}{\vec{p}}+\frac{1}{d}\right)} d z \\
& =\frac{2^{\frac{d}{2 p}-\frac{1}{2}}}{\frac{1}{2}-\frac{d}{2 p}+\frac{d}{2 s}}+\frac{2^{\frac{d}{2 p}-\frac{1}{2}}}{1-\frac{d}{2 s}}+\frac{2^{\frac{d}{2 s}-\frac{1}{2}+\frac{d}{2 p}-\frac{d}{2 \widetilde{p}}}}{\frac{1}{2}-\frac{d}{2 p}+\frac{d}{2 \widetilde{p}}}+\frac{2^{\frac{d}{2 s}-\frac{1}{2}+\frac{d}{2 p}-\frac{d}{2 \widetilde{p}}}}{1-\frac{d}{2 s}} \\
& :=M_{2 a} \text {. }
\end{aligned}
$$


If $t>1$ then

$$
\begin{aligned}
& G_{2}(t) \\
& =\int_{0}^{1 / 2}\left[\max \left\{(t-\tau)^{-\frac{d}{2}}, 1\right\}\right]^{\frac{1}{s}}\left(\left[\max \left\{\tau^{-\frac{d}{2}}, 1\right\}\right]^{\frac{1}{p}-\frac{1}{s}+\frac{1}{d}}+\left[\max \left\{\tau^{-\frac{d}{2}}, 1\right\}\right]^{\frac{1}{p}-\frac{1}{\widetilde{p}}+\frac{1}{d}}\right) e^{-\beta(t-\tau)} d \tau \\
& +\int_{1 / 2}^{1}\left[\max \left\{(t-\tau)^{-\frac{d}{2}}, 1\right\}\right]^{\frac{1}{s}}\left(\left[\max \left\{\tau^{-\frac{d}{2}}, 1\right\}\right]^{\frac{1}{p}-\frac{1}{s}+\frac{1}{d}}+\left[\max \left\{\tau^{-\frac{d}{2}}, 1\right\}\right]^{\frac{1}{p}-\frac{1}{\tilde{p}}+\frac{1}{d}}\right) e^{-\beta(t-\tau)} d \tau \\
& +\int_{1}^{t}\left[\max \left\{(t-\tau)^{-\frac{d}{2}}, 1\right\}\right]^{\frac{1}{s}}\left(\left[\max \left\{\tau^{-\frac{d}{2}}, 1\right\}\right]^{\frac{1}{p}-\frac{1}{s}+\frac{1}{d}}+\left[\max \left\{\tau^{-\frac{d}{2}}, 1\right\}\right]^{\frac{1}{p}-\frac{1}{\widetilde{p}}+\frac{1}{d}}\right) e^{-\beta(t-\tau)} d \tau \\
& \leq \int_{0}^{1 / 2}\left((t-\tau)^{-\frac{d}{2 s}}+1\right)\left(\tau^{-\frac{d}{2}\left(\frac{1}{p}-\frac{1}{s}+\frac{1}{d}\right)}+\tau^{-\frac{d}{2}\left(\frac{1}{p}-\frac{1}{\tilde{p}}+\frac{1}{d}\right)}\right) e^{-\beta(t-\tau)} d \tau \\
& +\int_{1 / 2}^{1}\left((t-\tau)^{-\frac{d}{2 s}}+1\right)\left(\tau^{-\frac{d}{2}\left(\frac{1}{p}-\frac{1}{s}+\frac{1}{d}\right)}+\tau^{-\frac{d}{2}\left(\frac{1}{p}-\frac{1}{\tilde{p}}+\frac{1}{d}\right)}\right) e^{-\beta(t-\tau)} d \tau \\
& +\int_{1}^{t}\left((t-\tau)^{-\frac{d}{2 s}}+1\right) 2 e^{-\beta(t-\tau)} d \tau \\
& \leq \int_{0}^{1 / 2}\left(2^{\frac{d}{2 s}}+1\right)\left(\tau^{-\frac{d}{2}\left(\frac{1}{p}-\frac{1}{s}+\frac{1}{d}\right)}+\tau^{-\frac{d}{2}\left(\frac{1}{p}-\frac{1}{\widetilde{p}}+\frac{1}{d}\right)}\right) d \tau \\
& +\int_{1 / 2}^{1}\left((t-\tau)^{-\frac{d}{2 s}}+1\right)\left(2^{\frac{d}{2}\left(\frac{1}{p}-\frac{1}{s}+\frac{1}{d}\right)}+2^{\frac{d}{2}\left(\frac{1}{p}-\frac{1}{\tilde{p}}+\frac{1}{d}\right)}\right) e^{-\beta(t-\tau)} d \tau \\
& +2 \int_{1}^{t}\left((t-\tau)^{-\frac{d}{2 s}}+1\right) e^{-\beta(t-\tau)} d \tau \\
& =\left(2^{\frac{d}{2 s}}+1\right)\left(\frac{2^{-\frac{1}{2}+\frac{d}{2 p}-\frac{d}{2 s}}}{\frac{1}{2}-\frac{d}{2 p}+\frac{d}{2 s}}+\frac{2^{-\frac{1}{2}+\frac{d}{2 p}-\frac{d}{2 \widetilde{p}}}}{\frac{1}{2}-\frac{d}{2 p}+\frac{d}{2 \widetilde{p}}}\right) \\
& +\left(2^{\frac{d}{2}\left(\frac{1}{p}-\frac{1}{s}+\frac{1}{d}\right)}+2^{\frac{d}{2}\left(\frac{1}{p}-\frac{1}{\tilde{p}}+\frac{1}{d}\right)}+2\right) \int_{-\infty}^{t}\left((t-\tau)^{-\frac{d}{2 s}}+1\right) e^{-\beta(t-\tau)} d \tau \\
& =\left(2^{\frac{d}{2 s}}+1\right)\left(\frac{2^{-\frac{1}{2}+\frac{d}{2 p}-\frac{d}{2 s}}}{\frac{1}{2}-\frac{d}{2 p}+\frac{d}{2 s}}+\frac{2^{-\frac{1}{2}+\frac{d}{2 p}-\frac{d}{2 \widetilde{p}}}}{\frac{1}{2}-\frac{d}{2 p}+\frac{d}{2 \widetilde{p}}}\right) \\
& +\left(2^{\frac{d}{2}\left(\frac{1}{p}-\frac{1}{s}+\frac{1}{d}\right)}+2^{\frac{d}{2}\left(\frac{1}{p}-\frac{1}{\widetilde{p}}+\frac{1}{d}\right)}+2\right)\left[\beta^{\left(\theta_{1}-1\right)} \Gamma\left(1-\theta_{1}\right)+\frac{1}{\beta}\right] \\
& :=M_{2 b},
\end{aligned}
$$

where $0<\theta_{1}:=\frac{d}{2 s}<1$. Therefore

$$
G_{2}(t) \leq M_{2}:=\max \left\{M_{2 a}, M_{2 b}\right\}
$$

Using the second inequality of Lemma 3.1, we obtain the estimates for $L^{\widetilde{p}}$-norm of the covariant derivative $\nabla u(t)$ as follows:

$$
\left[c_{d}(t)\right]^{-\left(\frac{1}{p}-\frac{1}{\tilde{p}}+\frac{1}{d}\right)}\|\nabla u(t)\|_{\widetilde{p}}
$$




$$
\begin{aligned}
\leq & C e^{-\beta t}\left(\left\|u_{0}\right\|_{p}+\left\|u_{0}\right\|_{2}\right) \\
& +\int_{0}^{t}\left[c_{d}(t)\right]^{-\left(\frac{1}{p}-\frac{1}{\tilde{p}}+\frac{1}{d}\right)}\left[c_{d}(t-\tau)\right]^{\frac{1}{p}-\frac{1}{\tilde{p}}+\frac{1}{d}} e^{-\beta(t-\tau)}\left(\|f(\tau)\|_{2}+\|f(\tau)\|_{p}\right) d \tau \\
& +\int_{0}^{t}\left[c_{d}(t)\right]^{-\left(\frac{1}{p}-\frac{1}{\widetilde{p}}+\frac{1}{d}\right)}\left[c_{d}(t-\tau)\right]^{\frac{1}{r}-\frac{1}{\widetilde{p}}+\frac{1}{d}} e^{-\beta(t-\tau)}\left(\left\|\nabla_{v} v(\tau)\right\|_{r}+\left\|\nabla_{v} v(\tau)\right\|_{2}\right) d \tau \\
\leq & C\left\|u_{0}\right\|_{L^{p} \cap L^{2}}+G_{31}(t)\|f\|_{\mathcal{X}} \\
& +\int_{0}^{t}\left[c_{d}(t)\right]^{-\left(\frac{1}{p}-\frac{1}{\tilde{p}}+\frac{1}{d}\right)}\left[c_{d}(t-\tau)\right]^{\frac{1}{r}-\frac{1}{\tilde{p}}+\frac{1}{d}} e^{-\beta(t-\tau)}\left(\|v(\tau)\|_{p}\|\nabla v(\tau)\|_{s}+\|v(\tau)\|_{p}\|\nabla v(\tau)\|_{\tilde{p}}\right) d \tau \\
\leq & C\left\|u_{0}\right\|_{L^{p} \cap L^{2}}+G_{31}(t)\|f\|_{\mathcal{X}} \\
& +\int_{0}^{t}\left[c_{d}(t)\right]^{-\left(\frac{1}{p}-\frac{1}{\tilde{p}}+\frac{1}{d}\right)}\left[c_{d}(t-\tau)\right]^{\frac{1}{r}-\frac{1}{\widetilde{p}}+\frac{1}{d}} e^{-\beta(t-\tau)}\left(\left[c_{d}(\tau)\right]^{\frac{1}{p}-\frac{1}{s}+\frac{1}{d}}+\left[c_{d}(\tau)\right]^{\frac{1}{p}-\frac{1}{\tilde{p}}+\frac{1}{d}}\right) d \tau\|v\|_{\mathcal{X}}^{2} \\
\leq & C\left\|u_{0}\right\|_{L^{p} \cap L^{2}}+G_{31}(t)\|f\|_{\mathcal{X}}+G_{32}(t)\|v\|_{\mathcal{X}}^{2} \leq C\left\|u_{0}\right\|_{L^{p} \cap L^{2}}+M_{31}\|f\|_{\mathcal{X}}+M_{32}\|v\|_{\mathcal{X}}^{2} \\
\leq & C_{2}\left(\left\|u_{0}\right\|_{L^{p} \cap L^{2}}+\|f\|_{\mathcal{X}}\right)+M_{32}\|v\|_{\mathcal{X}}^{2},
\end{aligned}
$$

where $C_{2}:=\max \left\{C, M_{31}\right\}$ and

$$
\begin{aligned}
G_{31} & =\int_{0}^{t}\left[c_{d}(t)\right]^{-\left(\frac{1}{p}-\frac{1}{\widetilde{p}}+\frac{1}{d}\right)}\left[c_{d}(t-\tau)\right]^{\frac{1}{p}-\frac{1}{\widetilde{p}}+\frac{1}{d}} e^{-\beta(t-\tau)} d \tau \leq M_{31}<+\infty \\
G_{32} & =\int_{0}^{t}\left[c_{d}(t)\right]^{-\left(\frac{1}{p}-\frac{1}{\tilde{p}}+\frac{1}{d}\right)}\left[c_{d}(t-\tau)\right]^{\frac{1}{r}-\frac{1}{\widetilde{p}}+\frac{1}{d}} e^{-\beta(t-\tau)}\left(\left[c_{d}(\tau)\right]^{\frac{1}{p}-\frac{1}{s}+\frac{1}{d}}+\left[c_{d}(\tau)\right]^{\frac{1}{p}-\frac{1}{\widetilde{p}}+\frac{1}{d}}\right) d \tau \\
& \leq M_{32}<+\infty
\end{aligned}
$$

We now estimate the integrals $G_{31}, G_{32}$ and give the corresponding precise values of $M_{31}$ and $M_{32}$ as follows:

$$
\begin{aligned}
G_{31}(t) & :=\int_{0}^{t}\left[c_{d}(t)\right]^{-\left(\frac{1}{p}-\frac{1}{\widetilde{p}}+\frac{1}{d}\right)}\left[c_{d}(t-\tau)\right]^{\frac{1}{p}-\frac{1}{\widetilde{p}}+\frac{1}{d}} e^{-\beta(t-\tau)} d \tau \\
& =\int_{0}^{t}\left[\max \left\{t^{-\frac{d}{2}}, 1\right\}\right]^{-\left(\frac{1}{p}-\frac{1}{\widetilde{p}}+\frac{1}{d}\right)}\left[\max \left\{(t-\tau)^{-\frac{d}{2}}, 1\right\}\right]^{\frac{1}{p}-\frac{1}{\widetilde{p}}+\frac{1}{d}} e^{-\beta(t-\tau)} d \tau
\end{aligned}
$$

If $0<t \leq 1$ then

$$
G_{31}(t) \leq \int_{0}^{t} t^{\frac{d}{2}\left(\frac{1}{p}-\frac{1}{\widetilde{p}}+\frac{1}{d}\right)}(t-\tau)^{-\frac{d}{2}\left(\frac{1}{p}-\frac{1}{\widetilde{p}}+\frac{1}{d}\right)} d \tau=\frac{t}{\frac{1}{2}-\frac{d}{2 p}+\frac{d}{2 \widetilde{p}}} \leq \frac{1}{\frac{1}{2}-\frac{d}{2 p}+\frac{d}{2 \widetilde{p}}}
$$

If $t>1$ then

$$
G_{31}(t) \leq \int_{0}^{t}\left[(t-\tau)^{-\frac{d}{2}\left(\frac{1}{p}-\frac{1}{\tilde{p}}+\frac{1}{d}\right)}+1\right] e^{-\beta(t-\tau)} d \tau \leq \beta^{\left(\theta_{31}-1\right)} \Gamma\left(1-\theta_{31}\right)+\frac{1}{\beta},
$$

where $0<\theta_{31}:=\frac{d}{2}\left(\frac{1}{p}-\frac{1}{\widetilde{p}}+\frac{1}{d}\right)<1$ and $M_{31}:=\max \left\{\frac{1}{\frac{1}{2}-\frac{d}{2 p}+\frac{d}{2 \widetilde{p}}}, \beta^{\left(\theta_{31}-1\right)} \Gamma\left(1-\theta_{31}\right)+\frac{1}{\beta}\right\}$. 
We continue to estimate the function $G_{32}(t)$ :

$$
\begin{aligned}
G_{32}(t)= & \int_{0}^{t}\left[c_{d}(t)\right]^{-\left(\frac{1}{p}-\frac{1}{\widetilde{p}}+\frac{1}{d}\right)}\left[c_{d}(t-\tau)\right]^{\frac{1}{r}-\frac{1}{\widetilde{p}}+\frac{1}{d}} e^{-\beta(t-\tau)}\left(\left[c_{d}(\tau)\right]^{\frac{1}{p}-\frac{1}{s}+\frac{1}{d}}+\left[c_{d}(\tau)\right]^{\frac{1}{p}-\frac{1}{\tilde{p}}+\frac{1}{d}}\right) d \tau \\
= & \int_{0}^{t}\left[\max \left\{t^{-\frac{d}{2}}, 1\right\}\right]^{-\left(\frac{1}{p}-\frac{1}{\widetilde{p}}+\frac{1}{d}\right)}\left[\max \left\{(t-\tau)^{-\frac{d}{2}}, 1\right\}\right]^{\frac{1}{r}-\frac{1}{\widetilde{p}}+\frac{1}{d}} \\
& \times\left(\left[\max \left\{\tau^{-\frac{d}{2}}, 1\right\}\right]^{\frac{1}{p}-\frac{1}{s}+\frac{1}{d}}+\left[\max \left\{\tau^{-\frac{d}{2}}, 1\right\}\right]^{\frac{1}{p}-\frac{1}{\tilde{p}}+\frac{1}{d}}\right) e^{-\beta(t-\tau)} d \tau .
\end{aligned}
$$

If $0<t \leq 1$ then

$$
\begin{aligned}
& G_{32}(t) \leq \int_{0}^{t} t^{\frac{d}{2}\left(\frac{1}{p}-\frac{1}{\tilde{p}}+\frac{1}{d}\right)}(t-\tau)^{-\frac{d}{2}\left(\frac{1}{r}-\frac{1}{\tilde{p}}+\frac{1}{d}\right)}\left(\tau^{-\frac{d}{2}\left(\frac{1}{p}-\frac{1}{s}+\frac{1}{d}\right)}+\tau^{-\frac{d}{2}\left(\frac{1}{p}-\frac{1}{\tilde{p}}+\frac{1}{d}\right)}\right) d \tau \\
& =\int_{0}^{t}\left(1-\frac{\tau}{t}\right)^{-\frac{d}{2}\left(\frac{1}{r}-\frac{1}{\widetilde{p}}+\frac{1}{d}\right)}\left(\frac{\tau}{t}\right)^{-\frac{d}{2}\left(\frac{1}{p}-\frac{1}{s}+\frac{1}{d}\right)} t^{\frac{1}{2}-\frac{d}{2 p}} d\left(\frac{\tau}{t}\right) \\
& +\int_{0}^{t}\left(1-\frac{\tau}{t}\right)^{-\frac{d}{2}\left(\frac{1}{r}-\frac{1}{\tilde{p}}+\frac{1}{d}\right)}\left(\frac{\tau}{t}\right)^{-\frac{d}{2}\left(\frac{1}{p}-\frac{1}{\tilde{p}}+\frac{1}{d}\right)} t^{\frac{1}{2}-\frac{d}{2}\left(\frac{1}{r}-\frac{1}{\tilde{p}}\right)} d\left(\frac{\tau}{t}\right) \\
& =\int_{0}^{1}(1-z)^{-\frac{d}{2}\left(\frac{1}{r}-\frac{1}{\tilde{p}}+\frac{1}{d}\right)} z^{-\frac{d}{2}\left(\frac{1}{p}-\frac{1}{s}+\frac{1}{d}\right)} t^{\frac{1}{2}-\frac{d}{2 p}} d z \\
& +\int_{0}^{1}(1-z)^{-\frac{d}{2}\left(\frac{1}{r}-\frac{1}{\tilde{p}}+\frac{1}{d}\right)} z^{-\frac{d}{2}\left(\frac{1}{p}-\frac{1}{\widetilde{p}}+\frac{1}{d}\right)} t^{\frac{1}{2}-\frac{d}{2}\left(\frac{1}{r}-\frac{1}{\widetilde{p}}\right)} d z \\
& \leq \int_{0}^{1 / 2} 2^{\frac{d}{2}\left(\frac{1}{r}-\frac{1}{\widetilde{p}}+\frac{1}{d}\right)} z^{-\frac{d}{2}\left(\frac{1}{p}-\frac{1}{s}+\frac{1}{d}\right)} d z+\int_{0}^{1 / 2} 2^{\frac{d}{2}\left(\frac{1}{r}-\frac{1}{\widetilde{p}}+\frac{1}{d}\right)} z^{-\frac{d}{2}\left(\frac{1}{p}-\frac{1}{\widetilde{p}}+\frac{1}{d}\right)} d z \\
& +\int_{1 / 2}^{1}(1-z)^{-\frac{d}{2}\left(\frac{1}{r}-\frac{1}{\widetilde{p}}+\frac{1}{d}\right)} 2^{\frac{d}{2}\left(\frac{1}{p}-\frac{1}{s}+\frac{1}{d}\right)} d z+\int_{1 / 2}^{1}(1-z)^{-\frac{d}{2}\left(\frac{1}{r}-\frac{1}{\tilde{p}}+\frac{1}{d}\right)} 2^{\frac{d}{2}\left(\frac{1}{p}-\frac{1}{\widetilde{p}}+\frac{1}{d}\right)} d z \\
& =\frac{2^{\frac{d}{p}-\frac{d}{2 \widetilde{p}}}}{\frac{1}{2}-\frac{d}{2 p}+\frac{d}{2 s}}+\frac{2^{\frac{d}{2 r}+\frac{d}{2 p}-\frac{d}{\widetilde{p}}}}{\frac{1}{2}-\frac{d}{2 p}+\frac{d}{2 \widetilde{p}}}+\frac{2^{\frac{d}{p}-\frac{d}{2 \widetilde{p}}}}{\frac{1}{2}+\frac{d}{2 \widetilde{p}}-\frac{d}{2 r}}+\frac{2^{\frac{d}{2 r}+\frac{d}{2 p}-\frac{d}{\widetilde{p}}}}{\frac{1}{2}+\frac{d}{2 \widetilde{p}}-\frac{d}{2 r}} \\
& :=M_{32 a} \text {. }
\end{aligned}
$$

If $t>1$ then

$$
\begin{aligned}
G_{32}(t)= & \int_{0}^{1 / 2}\left[\max \left\{t^{-\frac{d}{2}}, 1\right\}\right]^{-\left(\frac{1}{p}-\frac{1}{\tilde{p}}+\frac{1}{d}\right)}\left[\max \left\{(t-\tau)^{-\frac{d}{2}}, 1\right\}\right]^{\frac{1}{r}-\frac{1}{\widetilde{p}}+\frac{1}{d}} \\
& \times\left(\left[\max \left\{\tau^{-\frac{d}{2}}, 1\right\}\right]^{\frac{1}{p}-\frac{1}{s}+\frac{1}{d}}+\left[\max \left\{\tau^{-\frac{d}{2}}, 1\right\}\right]^{\frac{1}{p}-\frac{1}{\tilde{p}}+\frac{1}{d}}\right) e^{-\beta(t-\tau)} d \tau \\
& +\int_{1 / 2}^{1}\left[\max \left\{t^{-\frac{d}{2}}, 1\right\}\right]^{-\left(\frac{1}{p}-\frac{1}{\tilde{p}}+\frac{1}{d}\right)}\left[\max \left\{(t-\tau)^{-\frac{d}{2}}, 1\right\}\right]^{\frac{1}{r}-\frac{1}{\tilde{p}}+\frac{1}{d}} \\
& \times\left(\left[\max \left\{\tau^{-\frac{d}{2}}, 1\right\}\right]^{\frac{1}{p}-\frac{1}{s}+\frac{1}{d}}+\left[\max \left\{\tau^{-\frac{d}{2}}, 1\right\}\right]^{\frac{1}{p}-\frac{1}{\tilde{p}}+\frac{1}{d}}\right) e^{-\beta(t-\tau)} d \tau \\
& +\int_{1}^{t}\left[\max \left\{t^{-\frac{d}{2}}, 1\right\}\right]^{-\left(\frac{1}{p}-\frac{1}{\widetilde{p}}+\frac{1}{d}\right)}\left[\max \left\{(t-\tau)^{-\frac{d}{2}}, 1\right\}\right]^{\frac{1}{r}-\frac{1}{\tilde{p}}+\frac{1}{d}} \\
& \times\left(\left[\max \left\{\tau^{-\frac{d}{2}}, 1\right\}\right]^{\frac{1}{p}-\frac{1}{s}+\frac{1}{d}}+\left[\max \left\{\tau^{-\frac{d}{2}}, 1\right\}\right]^{\frac{1}{p}-\frac{1}{\tilde{p}}+\frac{1}{d}}\right) e^{-\beta(t-\tau)} d \tau
\end{aligned}
$$




$$
\begin{aligned}
& \leq \int_{0}^{1 / 2}\left[(t-\tau)^{-\frac{d}{2}\left(\frac{1}{r}-\frac{1}{\tilde{p}}+\frac{1}{d}\right)}+1\right]\left(\tau^{-\frac{d}{2}\left(\frac{1}{p}-\frac{1}{s}+\frac{1}{d}\right)}+\tau^{-\frac{d}{2}\left(\frac{1}{p}-\frac{1}{\tilde{p}}+\frac{1}{d}\right)}\right) e^{-\beta(t-\tau)} d \tau \\
& +\int_{1 / 2}^{1}\left[(t-\tau)^{-\frac{d}{2}\left(\frac{1}{r}-\frac{1}{\widetilde{p}}+\frac{1}{d}\right)}+1\right]\left(\tau^{-\frac{d}{2}\left(\frac{1}{p}-\frac{1}{s}+\frac{1}{d}\right)}+\tau^{-\frac{d}{2}\left(\frac{1}{p}-\frac{1}{\widetilde{p}}+\frac{1}{d}\right)}\right) e^{-\beta(t-\tau)} d \tau \\
& +\int_{1}^{t}\left[(t-\tau)^{-\frac{d}{2}\left(\frac{1}{r}-\frac{1}{\widetilde{p}}+\frac{1}{d}\right)}+1\right] 2 e^{-\beta(t-\tau)} d \tau \\
& \leq \int_{0}^{1 / 2}\left[2^{\frac{d}{2}\left(\frac{1}{r}-\frac{1}{\widetilde{p}}+\frac{1}{d}\right)}+1\right]\left(\tau^{-\frac{d}{2}\left(\frac{1}{p}-\frac{1}{s}+\frac{1}{d}\right)}+\tau^{-\frac{d}{2}\left(\frac{1}{p}-\frac{1}{\widetilde{p}}+\frac{1}{d}\right)}\right) e^{-\beta(t-\tau)} d \tau \\
& +\int_{1 / 2}^{1}\left[(t-\tau)^{-\frac{d}{2}\left(\frac{1}{r}-\frac{1}{\tilde{p}}+\frac{1}{d}\right)}+1\right]\left(2^{\frac{d}{2}\left(\frac{1}{p}-\frac{1}{s}+\frac{1}{d}\right)}+2^{\frac{d}{2}\left(\frac{1}{p}-\frac{1}{\tilde{p}}+\frac{1}{d}\right)}\right) e^{-\beta(t-\tau)} d \tau \\
& +\int_{1}^{t}\left[(t-\tau)^{-\frac{d}{2}\left(\frac{1}{r}-\frac{1}{\widetilde{p}}+\frac{1}{d}\right)}+1\right] 2 e^{-\beta(t-\tau)} d \tau \\
& \leq \int_{0}^{1 / 2}\left[2^{\frac{d}{2}\left(\frac{1}{r}-\frac{1}{\tilde{p}}+\frac{1}{d}\right)}+1\right]\left(\tau^{-\frac{d}{2}\left(\frac{1}{p}-\frac{1}{s}+\frac{1}{d}\right)}+\tau^{-\frac{d}{2}\left(\frac{1}{p}-\frac{1}{\tilde{p}}+\frac{1}{d}\right)}\right) d \tau \\
& +\int_{-\infty}^{t}(t-\tau)^{-\frac{d}{2}\left(\frac{1}{r}-\frac{1}{\tilde{p}}+\frac{1}{d}\right)}\left(2^{\frac{d}{2}\left(\frac{1}{p}-\frac{1}{s}+\frac{1}{d}\right)}+2^{\frac{d}{2}\left(\frac{1}{p}-\frac{1}{\tilde{p}}+\frac{1}{d}\right)}+2\right) e^{-\beta(t-\tau)} d \tau \\
& +\int_{-\infty}^{t}\left(2^{\frac{d}{2}\left(\frac{1}{p}-\frac{1}{s}+\frac{1}{d}\right)}+2^{\frac{d}{2}\left(\frac{1}{p}-\frac{1}{\tilde{p}}+\frac{1}{d}\right)}+2\right) e^{-\beta(t-\tau)} d \tau \\
& \leq\left[2^{\frac{d}{2}\left(\frac{1}{r}-\frac{1}{\widetilde{p}}+\frac{1}{d}\right)}+1\right]\left[\frac{2^{-\frac{1}{2}+\frac{d}{2 p}-\frac{d}{2 s}}}{\frac{1}{2}-\frac{d}{2 p}+\frac{d}{2 s}}+\frac{2^{-\frac{1}{2}+\frac{d}{2 p}-\frac{d}{2 \widetilde{p}}}}{\frac{1}{2}-\frac{d}{2 p}+\frac{d}{2 \widetilde{p}}}\right] \\
& +\left(2^{\frac{d}{2}\left(\frac{1}{p}-\frac{1}{s}+\frac{1}{d}\right)}+2^{\frac{d}{2}\left(\frac{1}{p}-\frac{1}{\tilde{p}}+\frac{1}{d}\right)}+2\right)\left(\beta^{\theta_{32}-1} \Gamma\left(1-\theta_{32}\right)+\frac{1}{\beta}\right) \\
& :=M_{32 b},
\end{aligned}
$$

where $0<\theta_{32}:=\frac{d}{2}\left(\frac{1}{r}-\frac{1}{\widetilde{p}}+\frac{1}{d}\right)<1$. Therefore $G_{32}(t) \leq M_{32}:=\max \left\{M_{32 a}, M_{32 b}\right\}$.

Using again the second inequality in Lemma 3.1, we obtain the estimates for $L^{s}$-norm of the covariant derivative $\nabla u(t)$ as follows:

$$
\begin{aligned}
& {\left[c_{d}(t)\right]^{-\left(\frac{1}{p}-\frac{1}{s}+\frac{1}{d}\right)}\|\nabla u(t)\|_{s} } \\
\leq & C e^{-\beta t}\left(\left\|u_{0}\right\|_{p}+\left\|u_{0}\right\|_{2}\right) \\
& +\int_{0}^{t}\left[c_{d}(t)\right]^{-\left(\frac{1}{p}-\frac{1}{s}+\frac{1}{d}\right)}\left[c_{d}(t-\tau)\right]^{-\left(\frac{1}{p}-\frac{1}{s}+\frac{1}{d}\right)} e^{-\beta(t-\tau)}\left(\|f(\tau)\|_{2}+\|f(\tau)\|_{p}\right) d \tau \\
& +\int_{0}^{t}\left[c_{d}(t)\right]^{-\left(\frac{1}{p}-\frac{1}{s}+\frac{1}{d}\right)}\left[c_{d}(t-\tau)\right]^{\frac{1}{r}-\frac{1}{s}+\frac{1}{d}} e^{-\beta(t-\tau)}\left(\left\|\nabla_{v} v(\tau)\right\|_{r}+\left\|\nabla_{v} v(\tau)\right\|_{2}\right) d \tau \\
\leq & C\left\|u_{0}\right\|_{L^{p} \cap L^{2}}+G_{41}(t)\|f\|_{\mathcal{X}} \\
& +\int_{0}^{t}\left[c_{d}(t)\right]^{-\left(\frac{1}{p}-\frac{1}{s}+\frac{1}{d}\right)}\left[c_{d}(t-\tau)\right]^{\frac{1}{s}+\frac{1}{d}} e^{-\beta(t-\tau)}\left(\|v(\tau)\|_{p}\|\nabla v(\tau)\|_{s}+\|v(\tau)\|_{p}\|\nabla v(\tau)\|_{\tilde{p}}\right) d \tau \\
\leq & C\left\|u_{0}\right\|_{L^{p} \cap L^{2}}+G_{41}(t)\|f\|_{\mathcal{X}}
\end{aligned}
$$




$$
\begin{aligned}
& +\int_{0}^{t}\left[c_{d}(t)\right]^{-\left(\frac{1}{p}-\frac{1}{s}+\frac{1}{d}\right)}\left[c_{d}(t-\tau)\right]^{\frac{1}{s}+\frac{1}{d}}\left(\left[c_{d}(\tau)\right]^{\frac{1}{p}-\frac{1}{s}+\frac{1}{d}}+\left[c_{d}(\tau)\right]^{\frac{1}{p}-\frac{1}{\tilde{p}}+\frac{1}{d}}\right) e^{-\beta(t-\tau)} d \tau\|v\|_{\mathcal{X}}^{2} \\
\leq & C\left\|u_{0}\right\|_{L^{p} \cap L^{2}}+G_{41}(t)\|f\|_{\mathcal{X}}+G_{42}(t)\|v\|_{\mathcal{X}}^{2} \\
\leq & C\left\|u_{0}\right\|_{L^{p} \cap L^{2}}+M_{41}\|f\|_{\mathcal{X}}+M_{42}\|v\|_{\mathcal{X}}^{2} \\
\leq & C_{3}\left(\left\|u_{0}\right\|_{L^{p} \cap L^{2}}+\|f\|_{\mathcal{X}}\right)+M_{42}\|v\|_{\mathcal{X}}^{2},
\end{aligned}
$$

where $C_{3}:=\max \left\{C, M_{41}\right\}$ and

$$
\begin{aligned}
G_{41} & =\int_{0}^{t}\left[c_{d}(t)\right]^{-\left(\frac{1}{p}-\frac{1}{s}+\frac{1}{d}\right)}\left[c_{d}(t-\tau)\right]^{-\left(\frac{1}{p}-\frac{1}{s}+\frac{1}{d}\right)} e^{-\beta(t-\tau)} d \tau \leq M_{41}<\infty \\
G_{42} & =\int_{0}^{t}\left[c_{d}(t)\right]^{-\left(\frac{1}{p}-\frac{1}{s}+\frac{1}{d}\right)}\left[c_{d}(t-\tau)\right]^{\frac{1}{s}+\frac{1}{d}}\left(\left[c_{d}(\tau)\right]^{\frac{1}{p}-\frac{1}{s}+\frac{1}{d}}+\left[c_{d}(\tau)\right]^{\frac{1}{p}-\frac{1}{\tilde{p}}+\frac{1}{d}}\right) e^{-\beta(t-\tau)} d \tau \\
& \leq M_{42}<\infty
\end{aligned}
$$

We then estimate the integrals $G_{41}, G_{42}$ and give the precise values of $M_{41}, M_{42}$ as follows:

$$
\begin{aligned}
& G_{41}(t):=\int_{0}^{t}\left[c_{d}(t)\right]^{-\left(\frac{1}{p}-\frac{1}{s}+\frac{1}{d}\right)}\left[c_{d}(t-\tau)\right]^{\frac{1}{p}-\frac{1}{s}+\frac{1}{d}} e^{-\beta(t-\tau)} d \tau
\end{aligned}
$$

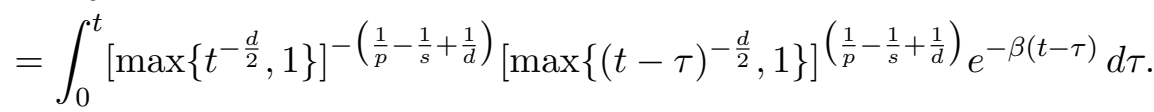

If $0<t \leq 1$ then

$$
G_{41}(t) \leq \int_{0}^{t} t^{\frac{d}{2}\left(\frac{1}{p}-\frac{1}{s}+\frac{1}{d}\right)}(t-\tau)^{-\frac{d}{2}\left(\frac{1}{p}-\frac{1}{s}+\frac{1}{d}\right)} d \tau=\frac{t}{\frac{1}{2}-\frac{d}{2 p}+\frac{d}{2 s}} \leq \frac{1}{\frac{1}{2}-\frac{d}{2 p}+\frac{d}{2 s}} .
$$

If $t>1$ then

$$
G_{41}(t) \leq \int_{0}^{t}\left[(t-\tau)^{-\frac{d}{2}\left(\frac{1}{p}-\frac{1}{s}+\frac{1}{d}\right)}+1\right] e^{-\beta(t-\tau)} d \tau \leq \beta^{\left(\theta_{41}-1\right)} \Gamma\left(1-\theta_{41}\right)+\frac{1}{\beta} \leq M_{41}
$$

where $0<\theta_{41}:=\frac{d}{2}\left(\frac{1}{p}-\frac{1}{s}+\frac{1}{d}\right)<1$ and

$$
M_{41}:=\max \left\{\frac{1}{\frac{1}{2}-\frac{d}{2 p}+\frac{d}{2 s}}, \beta^{\left(\theta_{41}-1\right)} \Gamma\left(1-\theta_{41}\right)+\frac{1}{\beta}\right\} .
$$

Next, we have

$$
\begin{aligned}
G_{42}(t)= & \int_{0}^{t}\left[c_{d}(t)\right]^{-\left(\frac{1}{p}-\frac{1}{s}+\frac{1}{d}\right)}\left[c_{d}(t-\tau)\right]^{\frac{1}{s}+\frac{1}{d}} e^{-\beta(t-\tau)}\left(\left[c_{d}(\tau)\right]^{\frac{1}{p}-\frac{1}{s}+\frac{1}{d}}+\left[c_{d}(\tau)\right]^{\frac{1}{p}-\frac{1}{\tilde{p}}+\frac{1}{d}}\right) d \tau \\
= & \int_{0}^{t}\left[\max \left\{t^{-\frac{d}{2}}, 1\right\}\right]^{-\left(\frac{1}{p}-\frac{1}{s}+\frac{1}{d}\right)}\left[\max \left\{(t-\tau)^{-\frac{d}{2}}, 1\right\}\right]^{\frac{1}{s}+\frac{1}{d}} \\
& \times\left(\left[\max \left\{\tau^{-\frac{d}{2}}, 1\right\}\right]^{\frac{1}{p}-\frac{1}{s}+\frac{1}{d}}+\left[\max \left\{\tau^{-\frac{d}{2}}, 1\right\}\right]^{\frac{1}{p}-\frac{1}{\tilde{p}}+\frac{1}{d}}\right) e^{-\beta(t-\tau)} d \tau
\end{aligned}
$$


If $0<t \leq 1$ then

$$
\begin{aligned}
& G_{42}(t) \leq \int_{0}^{t} t^{\frac{d}{2}\left(\frac{1}{p}-\frac{1}{s}+\frac{1}{d}\right)}(t-\tau)^{-\frac{d}{2}\left(\frac{1}{s}+\frac{1}{d}\right)}\left(\tau^{-\frac{d}{2}\left(\frac{1}{p}-\frac{1}{s}+\frac{1}{d}\right)}+\tau^{-\frac{d}{2}\left(\frac{1}{p}-\frac{1}{\tilde{p}}+\frac{1}{d}\right)}\right) d \tau \\
& =\int_{0}^{t}\left(1-\frac{\tau}{t}\right)^{-\frac{d}{2}\left(\frac{1}{s}+\frac{1}{d}\right)}\left(\frac{\tau}{t}\right)^{-\frac{d}{2}\left(\frac{1}{p}-\frac{1}{s}+\frac{1}{d}\right)} t^{\frac{1}{2}-\frac{d}{2 s}} d\left(\frac{\tau}{t}\right) \\
& +\int_{0}^{t}\left(1-\frac{\tau}{t}\right)^{-\frac{d}{2}\left(\frac{1}{s}+\frac{1}{d}\right)}\left(\frac{\tau}{t}\right)^{-\frac{d}{2}\left(\frac{1}{p}-\frac{1}{\tilde{p}}+\frac{1}{d}\right)} t^{\frac{1}{2}+\frac{d}{2 \widetilde{p}}-\frac{d}{2 s}} d\left(\frac{\tau}{t}\right) \\
& =\int_{0}^{1}(1-z)^{-\frac{d}{2}\left(\frac{1}{s}+\frac{1}{d}\right)} z^{-\frac{d}{2}\left(\frac{1}{p}-\frac{1}{s}+\frac{1}{d}\right)} t^{\frac{1}{2}-\frac{d}{2 s}} d z \\
& +\int_{0}^{1}(1-z)^{-\frac{d}{2}\left(\frac{1}{s}+\frac{1}{d}\right)} z^{-\frac{d}{2}\left(\frac{1}{p}-\frac{1}{\tilde{p}}+\frac{1}{d}\right)} t^{\frac{1}{2}+\frac{d}{2 \widetilde{p}}-\frac{d}{2 s}} d z \\
& \leq \int_{0}^{1 / 2} 2^{\frac{d}{2}\left(\frac{1}{s}+\frac{1}{d}\right)} z^{-\frac{d}{2}\left(\frac{1}{p}-\frac{1}{s}+\frac{1}{d}\right)} d z+\int_{0}^{1 / 2} 2^{\frac{d}{2}\left(\frac{1}{s}+\frac{1}{d}\right)} z^{-\frac{d}{2}\left(\frac{1}{p}-\frac{1}{\widetilde{p}}+\frac{1}{d}\right)} d z \\
& +\int_{1 / 2}^{1}(1-z)^{-\frac{d}{2}\left(\frac{1}{s}+\frac{1}{d}\right)} 2^{\frac{d}{2}\left(\frac{1}{p}-\frac{1}{s}+\frac{1}{d}\right)} d z+\int_{1 / 2}^{1}(1-z)^{-\frac{d}{2}\left(\frac{1}{s}+\frac{1}{d}\right)} 2^{\frac{d}{2}\left(\frac{1}{p}-\frac{1}{\widetilde{p}}+\frac{1}{d}\right)} d z \\
& =\frac{2^{\frac{d}{2 p}}}{\frac{1}{2}-\frac{d}{2 p}+\frac{d}{2 s}}+\frac{2^{\frac{d}{2 r}-\frac{d}{2 \widetilde{p}}}}{\frac{1}{2}-\frac{d}{2 p}+\frac{d}{2 \widetilde{p}}}+\frac{2^{\frac{d}{2 p}}}{\frac{1}{2}-\frac{d}{2 s}}+\frac{2^{\frac{d}{2 r}-\frac{d}{2 \widetilde{p}}}}{\frac{1}{2}-\frac{d}{2 s}} \\
& :=M_{42 a} \text {. }
\end{aligned}
$$

If $t>1$ then

$$
\begin{aligned}
G_{42}(t)= & \int_{0}^{1 / 2}\left[\max \left\{t^{-\frac{d}{2}}, 1\right\}\right]^{-\left(\frac{1}{p}-\frac{1}{s}+\frac{1}{d}\right)}\left[\max \left\{(t-\tau)^{-\frac{d}{2}}, 1\right\}\right]^{\frac{1}{s}+\frac{1}{d}} \\
& \times\left(\left[\max \left\{\tau^{-\frac{d}{2}}, 1\right\}\right]^{\frac{1}{p}-\frac{1}{s}+\frac{1}{d}}+\left[\max \left\{\tau^{-\frac{d}{2}}, 1\right\}\right]^{\frac{1}{p}-\frac{1}{\widetilde{p}}+\frac{1}{d}}\right) e^{-\beta(t-\tau)} d \tau \\
& +\int_{1 / 2}^{1}\left[\max \left\{t^{-\frac{d}{2}}, 1\right\}\right]^{-\left(\frac{1}{p}-\frac{1}{s}+\frac{1}{d}\right)}\left[\max \left\{(t-\tau)^{-\frac{d}{2}}, 1\right\}\right]^{\frac{1}{s}+\frac{1}{d}} \\
& \times\left(\left[\max \left\{\tau^{-\frac{d}{2}}, 1\right\}\right]^{\frac{1}{p}-\frac{1}{s}+\frac{1}{d}}+\left[\max \left\{\tau^{-\frac{d}{2}}, 1\right\}\right]^{\frac{1}{p}-\frac{1}{\widetilde{p}}+\frac{1}{d}}\right) e^{-\beta(t-\tau)} d \tau \\
& +\int_{1}^{t}\left[\max \left\{t^{-\frac{d}{2}}, 1\right\}\right]^{-\left(\frac{1}{p}-\frac{1}{s}+\frac{1}{d}\right)}\left[\max \left\{(t-\tau)^{-\frac{d}{2}}, 1\right\}\right]^{\frac{1}{s}+\frac{1}{d}} \\
& \times\left(\left[\max \left\{\tau^{-\frac{d}{2}}, 1\right\}\right]^{\frac{1}{p}-\frac{1}{s}+\frac{1}{d}}+\left[\max \left\{\tau^{-\frac{d}{2}}, 1\right\}\right]^{\frac{1}{p}-\frac{1}{\tilde{p}}+\frac{1}{d}}\right) e^{-\beta(t-\tau)} d \tau \\
\leq & \int_{0}^{1 / 2}\left[(t-\tau)^{-\frac{d}{2}\left(\frac{1}{s}+\frac{1}{d}\right)}+1\right]\left(\tau^{-\frac{d}{2}\left(\frac{1}{p}-\frac{1}{s}+\frac{1}{d}\right)}+\tau^{-\frac{d}{2}\left(\frac{1}{p}-\frac{1}{\tilde{p}}+\frac{1}{d}\right)}\right) e^{-\beta(t-\tau)} d \tau \\
+ & \int_{1 / 2}^{1}\left[(t-\tau)^{-\frac{d}{2}\left(\frac{1}{s}+\frac{1}{d}\right)}+1\right]\left(\tau^{-\frac{d}{2}\left(\frac{1}{p}-\frac{1}{s}+\frac{1}{d}\right)}+\tau^{-\frac{d}{2}\left(\frac{1}{p}-\frac{1}{\left.\widetilde{p}+\frac{1}{d}\right)}\right)} e^{-\beta(t-\tau)} d \tau\right. \\
& +\int_{1}^{t}\left[(t-\tau)^{-\frac{d}{2}\left(\frac{1}{s}+\frac{1}{d}\right)}+1\right] 2 e^{-\beta(t-\tau)} d \tau \\
\leq & \int_{0}^{1 / 2}\left(2^{\frac{d}{2}\left(\frac{1}{s}+\frac{1}{d}\right)}+1\right)\left(\tau^{-\frac{d}{2}\left(\frac{1}{p}-\frac{1}{s}+\frac{1}{d}\right)}+\tau^{-\frac{d}{2}\left(\frac{1}{p}-\frac{1}{\tilde{p}}+\frac{1}{d}\right)}\right) e^{-\beta(t-\tau)} d \tau
\end{aligned}
$$




$$
\begin{aligned}
& +\int_{1 / 2}^{1}\left[(t-\tau)^{-\frac{d}{2}\left(\frac{1}{s}+\frac{1}{d}\right)}+1\right]\left(2^{\frac{d}{2}\left(\frac{1}{p}-\frac{1}{s}+\frac{1}{d}\right)}+2^{\frac{d}{2}\left(\frac{1}{p}-\frac{1}{\widetilde{p}}+\frac{1}{d}\right)}\right) e^{-\beta(t-\tau)} d \tau \\
& +\int_{1}^{t}\left[(t-\tau)^{-\frac{d}{2}\left(\frac{1}{s}+\frac{1}{d}\right)}+1\right] 2 e^{-\beta(t-\tau)} d \tau \\
\leq & \int_{0}^{1 / 2}\left(2^{\frac{d}{2}\left(\frac{1}{s}+\frac{1}{d}\right)}+1\right)\left(\tau^{-\frac{d}{2}\left(\frac{1}{p}-\frac{1}{s}+\frac{1}{d}\right)}+\tau^{-\frac{d}{2}\left(\frac{1}{p}-\frac{1}{\widetilde{p}}+\frac{1}{d}\right)}\right) d \tau \\
& +\int_{-\infty}^{t}(t-\tau)^{-\frac{d}{2}\left(\frac{1}{s}+\frac{1}{d}\right)}\left(2^{\frac{d}{2}\left(\frac{1}{p}-\frac{1}{s}+\frac{1}{d}\right)}+2^{\frac{d}{2}\left(\frac{1}{p}-\frac{1}{\widetilde{p}}+\frac{1}{d}\right)}+2\right) e^{-\beta(t-\tau)} d \tau \\
& +\int_{-\infty}^{t}\left(2^{\frac{d}{2}\left(\frac{1}{p}-\frac{1}{s}+\frac{1}{d}\right)}+2^{\frac{d}{2}\left(\frac{1}{p}-\frac{1}{\tilde{p}}+\frac{1}{d}\right)}+2\right) e^{-\beta(t-\tau)} d \tau \\
\leq & \left(2^{\frac{d}{2}\left(\frac{1}{s}+\frac{1}{d}\right)}+1\right)\left(\frac{2^{-\frac{1}{2}+\frac{d}{2 p}-\frac{d}{2 s}}}{\frac{1}{2}-\frac{d}{2 p}+\frac{d}{2 s}}+\frac{2^{-\frac{1}{2}+\frac{d}{2 p}-\frac{d}{2 \widetilde{p}}}}{\frac{1}{2}-\frac{d}{2 p}+\frac{d}{2 \widetilde{p}}}\right) \\
& +\left(2^{\frac{d}{2}\left(\frac{1}{p}-\frac{1}{s}+\frac{1}{d}\right)}+2^{\frac{d}{2}\left(\frac{1}{p}-\frac{1}{\widetilde{p}}+\frac{1}{d}\right)}+2\right)\left(\beta^{\theta_{42}-1} \Gamma\left(1-\theta_{42}\right)+\frac{1}{\beta}\right) \\
:= & M_{42 b} .
\end{aligned}
$$

Putting $0<\theta_{42}:=\frac{d}{2}\left(\frac{1}{s}+\frac{1}{d}\right)<1$ and $M_{42}:=\max \left\{M_{42 a}, M_{42 b}\right\}$, we obtain $G_{42}(t) \leq M_{42}$.

Finally, the inequality 3.2 holds if we take

$$
\widetilde{C}=\max \left\{C_{1}, C_{2}, C_{3}\right\} \quad \text { and } \quad \widetilde{M}=\max \left\{M_{1}, M_{2}, M_{32}, M_{42}\right\} .
$$

We now invoke the Massera principle to prove Theorem 1.2 on the existence and uniqueness of a mild $T$-periodic solution to the Stokes equations (1.3).

Proof of Theorem 1.2. For each initial data $x \in L^{p}(\Gamma(T \mathcal{M})) \cap L^{2}(\Gamma(T \mathcal{M}))$ from Lemma 3.2 . there exists a unique mild solution $u \in \mathcal{X}$ to 1.3 with $u(0)=x$. This fact allows to define the Poincaré map $\mathcal{P}: L^{p}(\Gamma(T \mathcal{M})) \cap L^{2}(\Gamma(T \mathcal{M})) \rightarrow L^{p}(\Gamma(T \mathcal{M})) \cap L^{2}(\Gamma(T \mathcal{M}))$ as follows: For each $x \in L^{p}(\Gamma(T \mathcal{M})) \cap L^{2}(\Gamma(T \mathcal{M}))$ we set

$$
\mathcal{P}(x):=u(T) \text { where } u \in \mathcal{X} \text { is the unique mild solution of } 1.3 \text { with } u(0)=x \text {. }
$$

Note that using the formula 1.5 of the solutions we have

$$
\mathcal{P}(x)=u(T)=e^{-T \mathcal{A}} x+\int_{0}^{T} e^{-(T-\tau) \mathcal{A}} \mathbb{P}\left[\nabla_{v} v(\tau)+f(\tau)\right] d \tau
$$

with $u$ as in 3.3 . Next, from $T$-periodicity of $v$ and $f$, we obtain

$$
\begin{aligned}
u((k+1) T) & =e^{-(k+1) T \mathcal{A}} u(0)+\int_{0}^{(k+1) T} e^{-((k+1) T-\tau) \mathcal{A}_{1}} \mathbb{P}\left[\nabla_{v} v(\tau)+f(\tau)\right] d \tau \\
& =e^{-(k+1) T \mathcal{A}} u(0)+\int_{0}^{k T} e^{-((k+1) T-\tau) \mathcal{A}} \mathbb{P}\left[\nabla_{v} v(\tau)+f(\tau)\right] d \tau
\end{aligned}
$$




$$
\begin{aligned}
& +\int_{k T}^{(k+1) T} e^{-((k+1) T-\tau) \mathcal{A}} \mathbb{P}\left[\nabla_{v} v(\tau)+f(\tau)\right] d \tau \\
= & e^{-T \mathcal{A}} e^{-k T \mathcal{A}} u(0)+\int_{0}^{k T} e^{-T \mathcal{A}} e^{-(k T-s) \mathcal{A}} \mathbb{P}\left[\nabla_{v} v(\tau)+f(\tau)\right] d \tau \\
& +\int_{0}^{T} e^{-(T-\tau) \mathcal{A}} \mathbb{P}\left[\nabla_{v} v(\tau)+f(\tau)\right] d \tau \\
= & e^{-T \mathcal{A}} e^{-k T \mathcal{A}} u(0)+\int_{0}^{k T} e^{-T \mathcal{A}} e^{-(k T-\tau) \mathcal{A}} \mathbb{P}\left[\nabla_{v} v(\tau)+f(\tau)\right] d \tau \\
& +\int_{0}^{T} e^{-(T-\tau) \mathcal{A}} \mathbb{P}\left[\nabla_{v} v(\tau)+f(\tau)\right] d \tau \\
= & e^{-T \mathcal{A}} u(k T)+\int_{0}^{T} e^{-(T-\tau) \mathcal{A}_{\mathbb{P}}\left[\nabla_{v} v(\tau)+f(\tau)\right] d \tau \quad \text { for all } k \in \mathbb{N} .}
\end{aligned}
$$

It follows that $\mathcal{P}^{k}(x)=u(k T)$ for all $k \in \mathbb{N}$. Since $F \in \mathcal{X},\left\{\mathcal{P}^{k}(x)\right\}_{k \in \mathbb{N}}$ is a bounded sequence in $L^{p}(\Gamma(T \mathcal{M})) \cap L^{2}(\Gamma(T \mathcal{M}))$.

Next, as usual in the ergodic theory, for each $n \in \mathbb{N}$ we define the Cesàro sume $\mathbf{P}_{n}$ by

$$
\mathbf{P}_{n}:=\frac{1}{n} \sum_{k=1}^{n} \mathcal{P}^{k}: L^{p}(\Gamma(T \mathcal{M})) \cap L^{2}(\Gamma(T \mathcal{M})) \rightarrow L^{p}(\Gamma(T \mathcal{M})) \cap L^{2}(\Gamma(T \mathcal{M}))
$$

Starting from $x=0$ and using the inequality 3.2 we get

$$
\sup _{k \in \mathbb{N}}\left\|\mathcal{P}^{k}(0)\right\|_{L^{p} \cap L^{2}} \leq \widetilde{C}\|f\|_{L^{p} \cap L^{2}}+\widetilde{M}\|v\|_{\mathcal{X}}^{2}
$$

The boundedness of $\left\{\mathcal{P}^{k}(0)\right\}_{k \in \mathbb{N}}$ in $X$ implies that the sequence $\left\{\mathbf{P}_{n}(0)\right\}_{n \in \mathbb{N}}=\left\{\frac{1}{n} \sum_{k=1}^{n}\right.$ $\left.\mathcal{P}^{k}(0)\right\}_{n \in \mathbb{N}}$ is clearly a bounded sequence in $X$. By 3.6 we obtain

$$
\sup _{n \in \mathbb{N}}\left\|\mathbf{P}_{n}(0)\right\|_{L^{p} \cap L^{2}} \leq \widetilde{C}\|f\|_{L^{p} \cap L^{2}}+\widetilde{M}\|v\|_{\mathcal{X}}^{2} .
$$

Since $L^{p}(\Gamma(T \mathcal{M})) \cap L^{2}(\Gamma(T \mathcal{M}))$ has a separable pre-dual $L^{\frac{p}{p-1}}(\Gamma(T \mathcal{M}))+L^{2}(\Gamma(T \mathcal{M}))$, from Banach-Alaoglu's Theorem there exists a subsequence $\left\{\mathbf{P}_{n_{k}}(0)\right\}$ of $\left\{\mathbf{P}_{n}(0)\right\}$ such that

$$
\begin{gathered}
\left\{\mathbf{P}_{n_{k}}(0)\right\} \stackrel{\text { weak- }^{*}}{\longrightarrow} \widehat{x} \in L^{p}(\Gamma(T \mathcal{M})) \cap L^{2}(\Gamma(T \mathcal{M})) \\
\text { with }\|\widehat{x}\|_{L^{p} \cap L^{2}} \leq \widetilde{C}\|f\|_{L^{p} \cap L^{2}}+\widetilde{M}\|v\|_{\mathcal{X}}^{2} .
\end{gathered}
$$

By simple computations using formula 3.5 we obtain $\mathcal{P} \mathbf{P}_{n}(0)-\mathbf{P}_{n}(0)=\frac{1}{n}\left(\mathcal{P}^{n+1}(0)-\right.$ $\mathcal{P}(0))$. Since the sequence $\left\{\mathcal{P}^{n+1}(0)\right\}_{n \in \mathbb{N}}$ is bounded in $L^{p}(\Gamma(T \mathcal{M})) \cap L^{2}(\Gamma(T \mathcal{M}))$, we get

$$
\lim _{n \rightarrow \infty}\left(\mathcal{P} \mathbf{P}_{n}(0)-\mathbf{P}_{n}(0)\right)=\lim _{n \rightarrow \infty} \frac{1}{n}\left(\mathcal{P}^{n+1}(0)-\mathcal{P}(0)\right)=0
$$

strongly in $L^{p}(\Gamma(T \mathcal{M})) \cap L^{2}(\Gamma(T \mathcal{M}))$. Therefore, for the subsequence $\left\{\mathbf{P}_{n_{k}}(0)\right\}$ from (3.7) we have $\mathcal{P} \mathbf{P}_{n_{k}}(0)-\mathbf{P}_{n_{k}}(0) \stackrel{\text { weak-* }^{*}}{\longrightarrow} 0$. This limit, together with (3.7), implies that

$$
\mathcal{P} \mathbf{P}_{n_{k}}(0) \stackrel{\text { weak-*}^{*}}{\longrightarrow} \widehat{x} \in L^{p}(\Gamma(T \mathcal{M})) \cap L^{2}(\Gamma(T \mathcal{M})) .
$$


We now show that $\mathcal{P}(\widehat{x})=\widehat{x}$. To do this, using the formula (3.4) and denoting by $\langle\cdot, \cdot\rangle$ the dual pair between $L^{p}(\Gamma(T \mathcal{M})) \cap L^{2}(\Gamma(T \mathcal{M}))$ and $L^{\frac{p}{p-1}}(\Gamma(T \mathcal{M}))+L^{2}(\Gamma(T \mathcal{M}))$. Then, since $e^{-T \mathcal{A}^{\prime}}$ leaves $L^{\frac{p}{p-1}}(\Gamma(T \mathcal{M}))+L^{2}(\Gamma(T \mathcal{M}))$ invariant, for all $h \in L^{\frac{p}{p-1}}(\Gamma(T \mathcal{M}))+$ $L^{2}(\Gamma(T \mathcal{M}))$ we have

$$
\begin{aligned}
\left\langle\mathcal{P} \mathbf{P}_{n_{k}}(0), h\right\rangle & =\left\langle e^{-T \mathcal{A}} \mathbf{P}_{n_{k}}(0), h\right\rangle+\left\langle\int_{0}^{T} e^{-(T-\tau) \mathcal{A}} \mathbb{P}\left[\nabla_{v} v(\tau)+f(\tau)\right] d \tau, h\right\rangle \\
& =\left\langle\mathbf{P}_{n_{k}}(0), e^{-T \mathcal{A}^{\prime}} h\right\rangle+\left\langle\int_{0}^{T} e^{-(T-\tau) \mathcal{A}} \mathbb{P}\left[\nabla_{v} v(\tau)+f(\tau)\right] d \tau, h\right\rangle \\
& \stackrel{n_{k} \rightarrow \infty}{\longrightarrow}\left\langle\widehat{x}, e^{-T \mathcal{A}^{\prime}} h\right\rangle+\left\langle\int_{0}^{T} e^{-(T-\tau) \mathcal{A}} \mathbb{P}\left[\nabla_{v} v(\tau)+f(\tau)\right] d \tau, h\right\rangle \\
& =\left\langle e^{-T \mathcal{A}} \widehat{x}, h\right\rangle+\left\langle\int_{0}^{T} e^{-(T-\tau) \mathcal{A}} \mathbb{P}\left[\nabla_{v} v(\tau)+f(\tau)\right] d \tau, h\right\rangle \\
& =\langle\mathcal{P}(\widehat{x}), h\rangle .
\end{aligned}
$$

This yields that

$$
\mathcal{P} \mathbf{P}_{n_{k}}(0) \stackrel{\text { weak- }^{*}}{\longrightarrow} \mathcal{P} \widehat{x} \in L^{p}(\Gamma(T \mathcal{M})) \cap L^{2}(\Gamma(T \mathcal{M})) .
$$

It now follows from 3.8 and 3.9 that

$$
\mathcal{P}(\widehat{x})=\widehat{x}
$$

Taking now the element $\widehat{x} \in L^{p}(\Gamma(T \mathcal{M})) \cap L^{2}(\Gamma(T \mathcal{M}))$ as an initial condition, by Lemma 3.2 there exists a unique mild solution $\widehat{u}(\cdot) \in \mathcal{X}$ satisfying $\widehat{u}(0)=\widehat{x}$. From the definition of Poincaré map $\mathcal{P}$ we arrive at $\widehat{u}(0)=\widehat{u}(T)$. Therefore, the solution $\widehat{u}(t)$ is periodic with the period $T$. The inequality (1.1) now follows from the inequalities (3.2) and (3.7).

We now prove the uniqueness of the periodic mild solution. Let $\widehat{u}_{1}$ and $\widehat{u}_{2}$ be two $T$-periodic mild solutions to equation (1.3) which belong to $\mathcal{X}$. Then, putting $v=\widehat{u}_{1}-\widehat{u}_{2}$ we have that $v$ is $T$-periodic and, by the formula 1.5 ,

$$
v(t)=e^{-t \mathcal{A}}\left(\widehat{u}_{1}(0)-\widehat{u}_{2}(0)\right) \text { for } t>0 .
$$

The smoothing property (see (3.1)) implies that

$$
\lim _{t \rightarrow \infty}\|v(t)\|_{L^{p} \cap L^{2}}=0 .
$$

This fact, together with the periodicity of $v$, gives that $v(t)=0$ for all $t \geq 0$. This yields $\widehat{u}_{1}=\widehat{u}_{2}$.

We now prove Theorem 1.3 on the existence, uniqueness and stability of periodic solutions to Navier-Stokes equations 1.2 which was stated in Section 1. Actually, we combine the fixed point argument and the results obtained in Theorem 1.2 to prove the second main result which will be done as follows. 
Proof of Theorem 1.3. Let

$$
\mathcal{B}_{\rho}^{T}:=\left\{v \in \mathcal{X}: v \text { is } T \text {-periodic and }\|v\|_{\mathcal{X}} \leq \rho\right\}
$$

Consider the equation

$$
\partial_{t} u+\mathcal{A} u=\mathbb{P}\left[\nabla_{v} v+f\right]
$$

Then, for $v \in \mathcal{B}_{\rho}^{T}$ we set

$$
\Phi(v):=u \text { where } u \in \mathcal{X} \text { is the unique } T \text {-periodic mild solution to } 3.10 \text {. }
$$

We will prove that if $\rho$ and $\|F\|_{\mathcal{X}}$ are small enough, then the transformation $\Phi$ acts from $\mathcal{B}_{\rho}^{T}$ into itself and is a contraction. Indeed, taking any $v \in \mathcal{B}_{\rho}^{T}$, applying Theorem 1.2 for the right-hand side $\nabla_{v} v+f$, we obtain that for $v \in \mathcal{B}_{\rho}^{T}$ there exists a unique $T$-periodic mild solution $u$ to 3.10 satisfying

$$
\|\widehat{u}\|_{\mathcal{X}} \leq(C+1)\left(C\|f\|_{L^{p} \cap L^{2}}+M\|v\|_{\mathcal{X}}^{2}\right)
$$

Therefore, if $\rho$ and $\|f\|_{L^{p} \cap L^{2}}$ are small enough, the map $\Phi$ acts from $\mathcal{B}_{\rho}^{T}$ into itself. Then, by (1.5), we have the following representation of $\Phi$ :

$$
\Phi(v)(t)=e^{-t \mathcal{A}} u(0)+\int_{0}^{t} e^{-(t-\tau) \mathcal{A}} \mathbb{P}\left[\left(\nabla_{v} v+f\right)(\tau)\right] d \tau \quad \text { for } \Phi(v)=u .
$$

Furthermore, for $v_{1}, v_{2} \in \mathcal{B}_{\rho}^{T}$ by the representation 1.1 we obtain that $u:=\Phi\left(v_{1}\right)-$ $\Phi\left(v_{2}\right)$ is the unique $T$-periodic mild solution to the equation

$$
\partial_{t} u+\mathcal{A} u=\mathbb{P}\left[\nabla_{v_{1}} v_{1}-\nabla_{v_{2}} v_{2}\right]=\mathbb{P}\left[\nabla_{v_{1}-v_{2}} v_{1}+\nabla_{v_{2}}\left(v_{1}-v_{2}\right)\right]
$$

Thus, again by Theorem 1.2 in the case $f=0$ we arrive at

$$
\begin{aligned}
\left\|\Phi\left(v_{1}\right)-\Phi\left(v_{2}\right)\right\|_{\mathcal{X}} & \leq(C+1) M\left(\left\|v_{1}-v_{2}\right\| \mathcal{X}\left\|v_{1}\right\|_{\mathcal{X}}+\left\|v_{2}\right\|_{\mathcal{X}}\left\|v_{1}-v_{2}\right\|_{\mathcal{X}}\right) \\
& \leq 2(C+1) M \rho\left\|v_{1}-v_{2}\right\|_{\mathcal{X}}
\end{aligned}
$$

We hence obtain that if $\rho$ are sufficiently small, then $\Phi: \mathcal{B}_{\rho}^{T} \rightarrow \mathcal{B}_{\rho}^{T}$ is a contraction. Therefore, for these values of $\rho$ and $\|f\|_{L^{p} \cap L^{2}}$, there exists a unique fixed point $\widehat{u}$ of $\Phi$, and by the definition of $\Phi$, this function $\widehat{u}$ is the unique $T$-periodic mild solution to Navier-Stokes equation (1.2).

To prove the stability of such a periodic solution we need the cone inequality theorem which we now recall. To this purpose, we first introduce the following notion of a cone in a Banach space as follows: A closed subset $\mathcal{K}$ of a Banach space $W$ is called a cone if 
(i) $x_{0} \in \mathcal{K}$ implies $\lambda x_{0} \in \mathcal{K}$ for all $\lambda \geq 0$;

(ii) $x_{1}, x_{2} \in \mathcal{K}$ implies $x_{1}+x_{2} \in \mathcal{K}$;

(iii) $\pm x_{0} \in \mathcal{K}$ implies $x_{0}=0$.

Then, let a cone $\mathcal{K}$ be given in the Banach space $W$. For $x, y \in W$ we then write $x \leq y$ if $y-x \in \mathcal{K}$.

If the cone $\mathcal{K}$ is invariant under a linear operator $A$, then it is easy to see that $A$ preserves the inequality, i.e., $x \leq y$ implies $A x \leq A y$. Now, the following theorem of cone inequality is taken from [3, Theorem I.9.3].

Theorem 3.3 (Cone inequality). Suppose that $\mathcal{K}$ is a cone in a Banach space $W$ such that $\mathcal{K}$ is invariant under a bounded linear operator $A \in \mathcal{L}(W)$ having spectral radius $r_{A}<1$. For a vector $x \in W$ satisfying

$$
x \leq A x+z \quad \text { for some given } z \in W
$$

we have that it also satisfies the estimate $x \leq y$, where $y \in W$ is a solution of the equation $y=A y+z$.

Lastly, we prove the third main result as follows.

Proof of Theorem 1.4. To prove the stability of the periodic solution $\widehat{u}$, we let $u(t)$ be any bounded solution of equation (1.6) corresponding to the initial value $u_{0}:=u(0) \in B_{\frac{\rho}{2}}:=$ $\left\{v \in L^{p}(\Gamma(T \mathcal{M})) \cap L^{2}(\Gamma(T \mathcal{M})):\|v\|_{L^{p} \cap L^{2}} \leq \frac{\rho}{2}\right\}$. Then, we have

$$
u(t)-\widehat{u}(t)=e^{-t \mathcal{A}}(u(0)-\widehat{u}(0))+\int_{0}^{t} e^{-(t-\tau) \mathcal{A}} \mathbb{P}\left[\nabla_{u} u(\tau)-\nabla_{\widehat{u}} \widehat{u}(\tau)\right] d \tau, \quad \text { for } t \geq 0 .
$$

By the same way as in the proof of Lemma 3.2, we can prove that

$$
\begin{aligned}
\|u(t)-\widehat{u}(t)\| \mid \leq & N e^{-\beta t}\|u(0)-\widehat{u}(0)\|_{L^{p} \cap L^{2}} \\
& +2 \rho \int_{0}^{t}\left(\bar{G}_{1}(\tau)+\bar{G}_{2}(\tau)+\bar{G}_{32}(\tau)+\bar{G}_{42}(\tau)\right) e^{-\beta(t-\tau)}\|\|(u-\widehat{u})(\tau)\|\| d \tau,
\end{aligned}
$$

where $\bar{G}_{1}(\tau), \bar{G}_{2}(\tau), \bar{G}_{32}(\tau)$ and $\bar{G}_{42}(\tau)$ are the functions determined as

$$
\begin{aligned}
& \bar{G}_{1}(t):=\left[c_{d}(\tau)\right]^{\frac{1}{p}-\frac{1}{\widetilde{p}}+\frac{1}{d}} \\
& \bar{G}_{2}(t):=\left[c_{d}(t-\tau)\right]^{\frac{1}{s}}\left(\left[c_{d}(\tau)\right]^{\frac{1}{p}-\frac{1}{s}+\frac{1}{d}}+\left[c_{d}(\tau)\right]^{\frac{1}{p}-\frac{1}{\widetilde{p}}+\frac{1}{d}}\right), \\
& \bar{G}_{3}(t):=\left[c_{d}(t)\right]^{-\left(\frac{1}{p}-\frac{1}{\widetilde{p}}+\frac{1}{d}\right)}\left[c_{d}(t-\tau)\right]^{\frac{1}{r}-\frac{1}{\widetilde{p}}+\frac{1}{d}}\left(\left[c_{d}(\tau)\right]^{\frac{1}{p}-\frac{1}{s}+\frac{1}{d}}+\left[c_{d}(\tau)\right]^{\frac{1}{p}-\frac{1}{\widetilde{p}}+\frac{1}{d}}\right), \\
& \bar{G}_{4}(t):=\left[c_{d}(t)\right]^{-\left(\frac{1}{p}-\frac{1}{s}+\frac{1}{d}\right)}\left[c_{d}(t-\tau)\right]^{\frac{1}{s}+\frac{1}{d}}\left(\left[c_{d}(\tau)\right]^{\frac{1}{p}-\frac{1}{s}+\frac{1}{d}}+\left[c_{d}(\tau)\right]^{\frac{1}{p}-\frac{1}{\widetilde{p}}+\frac{1}{d}}\right) .
\end{aligned}
$$


Put $\phi(t):=|\|u(t)-\widehat{u}(t)\||$. Then $\sup _{t \geq 0} \phi(t)<\infty$, and for $t>0$,

$$
\begin{aligned}
\phi(t) \leq & N e^{-\beta t}\|u(0)-\widehat{u}(0)\|_{L^{p} \cap L^{2}} \\
& +2 \rho \int_{0}^{t}\left(\bar{G}_{1}(\tau)+\bar{G}_{2}(\tau)+\bar{G}_{32}(\tau)+\bar{G}_{42}(\tau)\right) e^{-\beta(t-\tau)} \phi(\tau) d \tau .
\end{aligned}
$$

We will use the Cone Inequality Theorem to Banach space $W:=L^{\infty}([0, \infty))$ which is the space of real-valued functions defined and essentially bounded on $[0, \infty)$ (endowed with the sup-norm denoted by $\|\cdot\|_{\infty}$ ) with the cone $\mathcal{K}$ being the set of all (a.e.) nonnegative functions. We now consider the linear operator $A$ defined for $h \in W$ by

$$
(A h)(t):=2 \rho \int_{0}^{t}\left(\bar{G}_{1}(\tau)+\bar{G}_{2}(\tau)+\bar{G}_{32}(\tau)+\bar{G}_{42}(\tau)\right) e^{-\beta(t-\tau)} h(\tau) d \tau \quad \text { for } t \geq 0 .
$$

Since the boundedness of the integral

$$
\int_{0}^{t}\left(\bar{G}_{1}(\tau)+\bar{G}_{2}(\tau)+\bar{G}_{32}(\tau)+\bar{G}_{42}(\tau)\right) e^{-\beta(t-\tau)} d \tau
$$

we have

$$
\sup _{t \geq 0}|(A h)(t)|=\sup _{t \geq 0} 2 \rho \int_{0}^{t}\left(\bar{G}_{1}(\tau)+\cdots+\bar{G}_{42}(\tau)\right) e^{-\beta(t-\tau)}|h(\tau)| d \tau \leq 2 \rho \bar{M}\|h\|_{\infty},
$$

where $\bar{M}:=M_{1}+M_{2}+M_{32}+M_{42}$ with the constants being defined as in Lemma 3.2 . Therefore, $A \in \mathcal{L}\left(L^{\infty}([0, \infty))\right)$ and $\|A\|_{\infty} \leq 2 \rho \bar{M}<1$ for $\rho$ being small enough. Note that if $\|u(0)-\widehat{u}(0)\|_{L^{p} \cap L^{2}}$ is small enough, by the same way as in the proof of (I) we can show that the solution $u(t)$ exists and unique in a small ball $\mathcal{B}_{\rho}$ of $\mathcal{X}$.

Obviously, $A$ leaves the cone $\mathcal{K}$ invariant. The inequality 3.11 can now be rewritten as

$$
\phi \leq A \phi+z \quad \text { for } z(t)=N e^{-\beta t}\|u(0)-\widehat{u}(0)\|_{L^{p} \cap L^{2}} ; t \geq 0 .
$$

Hence, by Theorem 3.3 we obtain that $\phi \leq \psi$, where $\psi$ is a solution in $L^{\infty}([0, \infty))$ of the equation $\psi=A \psi+z$ which can be rewritten as

$$
\begin{aligned}
\psi(t)= & N e^{-\beta t}\|u(0)-\widehat{u}(0)\|_{L^{p} \cap L^{2}} \\
& +2 \rho \int_{0}^{t}\left(\bar{G}_{1}(\tau)+\cdots+\bar{G}_{42}(\tau)\right) e^{-\beta(t-\tau)}|\psi(\tau)| d \tau \quad \text { for } t \geq 0 .
\end{aligned}
$$

In order to estimate $\psi$, for $0<\gamma<\beta$ we set $w(t):=e^{\gamma t} \psi(t), t \geq 0$. Then, by 3.12 we obtain that

$$
\begin{aligned}
w(t)= & N e^{-(\beta-\gamma) t}\|u(0)-\widehat{u}(0)\|_{L^{p} \cap L^{2}} \\
& +2 \rho \int_{0}^{t}\left(\bar{G}_{1}(\tau)+\cdots+\bar{G}_{42}(\tau)\right) e^{-(\beta-\gamma)(t-\tau)} w(\tau) d \tau \quad \text { for } t \geq 0 .
\end{aligned}
$$


We next consider the linear operator $D$ defined for $\varphi \in L^{\infty}([0, \infty))$ by

$$
(D \varphi)(t)=2 \rho \int_{0}^{t}\left(\bar{G}_{1}(\tau)+\cdots+\bar{G}_{42}(\tau)\right) e^{-(\beta-\gamma)(t-\tau)} \varphi(\tau) d \tau \quad \text { for } t \geq 0 .
$$

Again, we can estimate

$$
\sup _{t \geq 0}|(D \varphi)(t)|=\sup _{t \geq 0} 2 \rho \int_{0}^{t}\left(\bar{G}_{1}(\tau)+\cdots+\bar{G}_{42}(\tau)\right) e^{-(\beta-\gamma)(t-\tau)}|\varphi(\tau)| d \tau \leq 2 \rho \bar{M}_{\gamma}\|\varphi\|_{\infty},
$$

where $\bar{M}_{\gamma}$ is the function obtained by substituting $\beta$ by $\beta-\gamma$ in $\bar{M}$.

Therefore, $D \in \mathcal{L}\left(L^{\infty}([0, \infty))\right)$ and we choose $0<\gamma<\beta$ such that $2 \rho \bar{M}_{\gamma}<1$ (the existence of such a constant $\gamma$ is explained at the end of proof) then $\|D\| \leq 2 \rho \bar{M}_{\gamma}<1$. Equation (3.13) can now be rewritten as

$$
w=D w+z \quad \text { for } z(t)=N e^{-(\beta-\gamma) t}\|u(0)-\widehat{u}(0)\|_{L^{p} \cap L^{2}}, t \geq 0 .
$$

Therefore, the equation $w=D w+z$ is uniquely solvable in $L^{\infty}([0, \infty))$, and its solution is $w=(I-D)^{-1} z$. Hence, we obtain that

$$
\begin{aligned}
\|w\|_{\infty} & =\left\|(I-D)^{-1} z\right\|_{\infty} \leq\left\|(I-D)^{-1}\right\|\|z\|_{\infty} \\
& \leq \frac{N}{1-\|D\|}\|u(0)-\widehat{u}(0)\|_{L^{p} \cap L^{2}} \\
& \leq \frac{N}{1-2 \rho \bar{M}_{\gamma}}\|u(0)-\widehat{u}(0)\|_{L^{p} \cap L^{2}}:=C_{\gamma}\|u(0)-\widehat{u}(0)\|_{L^{p} \cap L^{2}},
\end{aligned}
$$

where $C_{\gamma}=\frac{N}{1-2 \rho \bar{M}_{\gamma}}$. This yields that

$$
w(t) \leq C_{\gamma}\|u(0)-\widehat{u}(0)\|_{L^{p} \cap L^{2}} \text { for } t \geq 0 .
$$

Hence, $\psi(t)=e^{-\gamma t} w(t) \leq C_{\gamma} e^{-\gamma t}\|u(0)-\widehat{u}(0)\|_{L^{p} \cap L^{2}}$. Since $\left.\| \mid u(t)-\widehat{u}(t)\right)\|\|=\phi(t) \leq \psi(t)$, we obtain that

$$
\|\| u(t)-\widehat{u}(t)\left\|\mid \leq C_{\gamma} e^{-\gamma t}\right\| u(0)-\widehat{u}(0) \|_{L^{p} \cap L^{2}} \quad \text { for } t \geq 0 .
$$

Now, it remains to show the existence of $\gamma$. Indeed, we have $\bar{M}_{\gamma}:=M_{1 \gamma}+M_{2 \gamma}+M_{32 \gamma}+$ $M_{42 \gamma}$, where $M_{1 \gamma}, M_{2 \gamma}, M_{32 \gamma}$ and $M_{42 \gamma}$ are the functions obtained by substituting $\beta$ by $\beta-\gamma$ in $M_{1}, M_{2}, M_{3}$ and $M_{42}$ respectively.

Setting $E$ the maximum of the terms which are independent of $\beta$ and $\gamma, \theta:=\max \left\{\theta_{1}\right.$, $\left.\theta_{32}, \theta_{42}\right\}$, and $F:=\max \left\{\Gamma\left(1-\theta_{1}\right), \Gamma\left(1-\theta_{32}\right), \Gamma\left(1-\theta_{42}\right)\right\}$, we have $E\left(7+\frac{3 F}{(\beta-\gamma)^{1-\theta}}+\right.$ $\left.\frac{3}{\beta-\gamma}\right)<\frac{1}{2 \rho}$. It follows that $\frac{3(F+1)}{(\beta-\gamma)^{1-\theta}}<\frac{1-14 E \rho}{2 E \rho}$. We obtain that $\gamma$ satisfies $0<\gamma<$ $\beta-\left(\frac{6 E(F+1) \rho}{1-14 E \rho}\right)^{\frac{1}{1-\theta}}$. The existence of $\gamma$ is guaranteed if $\rho$ is small enough due to the fact that function $\left(\frac{6 E(F+1) \rho}{1-14 E \rho}\right)^{\frac{1}{1-\theta}}$ is increasing on $\rho$ and it tends to zero as $\rho$ decreases to zero. Our proof is completed. 


\section{Acknowledgments}

The works of the first two authors and the last author were partially supported by Vietnam Institute for Advance Study in Mathematics (VIASM). The work of the last author is partly supported by by the Project of Vietnam Ministry of Education and Training under Project B2022-BKA-06. This work is financially supported by Vietnam National Foundation for Science and Technology Development (NAFOSTED) under Grant No. 101.022021.04 .

\section{References}

[1] C. H. Chan and M. Czubak, Remarks on the weak formulation of the Navier-Stokes equations on the 2D hyperbolic space, Ann. Inst. H. Poincaré Anal. Non Linéaire 33 (2016), no. 3, 655-698.

[2] C. H. Chan, M. Czubak and M. M. Disconzi, The formulation of the Navier-Stokes equations on Riemannian manifolds, J. Geom. Phys. 121 (2017), 335-346.

[3] Ju. L. Dalec'kiŭ and M. G. Kreı̆n, Stability of Solutions of Differential Equations in Banach Space, Translations of Mathematical Monographs 43, American Mathematical Society, Providence, R.I., 1974.

[4] E. Damek and F. Ricci, A class of nonsymmetric harmonic Riemannian spaces, Bull. Amer. Math. Soc. (N.S.) 27 (1992), no. 1, 139-142.

[5] P. B. Eberlein, Geometry of Nonpositively Curved Manifolds, Chicago Lectures in Mathematics, University of Chicago press, Chicago, IL, 1996.

[6] D. G. Ebin and J. Marsden, Groups of diffeomorphisms and the motion of an incompressible fluid, Ann. of Math. (2) 92 (1970), 102-163.

[7] G. P. Galdi, Existence and uniqueness of time-periodic solutions to the Navier-Stokes equations in the whole plane, Discrete Contin. Dyn. Syst. Ser. S 6 (2013), no. 5, $1237-1257$.

[8] _ On time-periodic flow of a viscous liquid past a moving cylinder, Arch. Ration. Mech. Anal. 210 (2013), no. 2, 451-498.

[9] G. P. Galdi and A. L. Silvestre, Existence of time-periodic solutions to the NavierStokes equations around a moving body, Pacific J. Math. 223 (2006), no. 2, 251-267.

[10] G. Galdi and H. Sohr, Existence and uniqueness of time-periodic physically reasonable Navier-Stokes flow past a body, Arch. Ration. Mech. Anal. 172 (2004), no. 3, 363-406. 
[11] M. Geissert, M. Hieber and T. H. Nguyen, A general approach to time periodic incompressible viscous fluid flow problems, Arch. Ration. Mech. Anal. 220 (2016), no. 3, 1095-1118.

[12] Y. Giga, Solutions for semilinear parabolic equations in $L^{p}$ and regularity of weak solutions of the Navier-Stokes system, J. Differential Equations 62 (1986), no. 2, $186-212$.

[13] E. Hebey, Nonlinear Analysis on Manifolds: Sobolev spaces and inequalities, Courant Lecture Notes in Mathematics 5, New York University, Courant Institute of Mathematical Sciences, New York, 1999.

[14] S. Helgason, Geometric Analysis on Symmetric Spaces, Mathematical Surveys and Monographs 39, American Mathematical Society, Providence, RI, 1994.

[15] J. G. Heywood, The Navier-Stokes equations: On the existence, regularity and decay of solutions, Indiana Univ. Math. J. 29 (1980), no. 5, 639-681.

[16] J. Jost, Riemannian Geometry and Geometric Analysis, Fifth edition, Universitext, Springer-Verlag, Berlin, 2008.

[17] S. Kaniel and M. Shinbrot, A reproductive property of the Navier-Stokes equations, Arch. Rational Mech. Anal. 24, (1967), 363-369.

[18] T. Kato, Strong $L^{p}$-solutions of the Navier-Stokes equation in $\mathbb{R}^{m}$ with applications to weak solutions, Math. Z. 187 (1984), no. 4, 471-480.

[19] B. Khesin and G. Misiołek, Euler and Navier-Stokes equations on the hyperbolic plane, Proc. Natl. Acad. Sci. USA 109 (2012), no. 45, 18324-18326.

[20] H. Kozono and M. Nakao, Periodic solutions of the Navier-Stokes equations in unbounded domains, Tohoku Math. J. (2) 48 (1996), no. 1, 33-50.

[21] L. A. Lichtenfelz, Nonuniqueness of solutions of the Navier-Stokes equations on Riemannian manifolds, Ann. Global Anal. Geom. 50 (2016), no. 3, 237-248.

[22] N. Lohoué, Estimation des projecteurs de de Rham Hodge de certaines variétés riemaniennes non compactes, Math. Nachr. 279 (2006), no. 3, 272-298.

[23] P. Maremonti, Existence and stability of time-periodic solutions to the Navier-Stokes equations in the whole space, Nonlinearity 4 (1991), no. 2, 503-529.

[24] P. Maremonti and M. Padula, Existence, uniqueness and attainability of periodic solutions of the Navier-Stokes equations in exterior domains, J. Math. Sci. (New York) 93 (1999), no. 5, 719-746. 
[25] T. Miyakawa and Y. Teramoto, Existence and periodicity of weak solutions of the Navier-Stokes equations in a time dependent domain, Hiroshima Math. J. 12 (1982), no. $3,513-528$.

[26] T. H. Nguyen, Periodic motions of Stokes and Navier-Stokes flows around a rotating obstacle, Arch. Ration. Mech. Anal. 213 (2014), no. 2, 689-703.

[27] T. H. Nguyen, T. X. Pham, T. N. H. Vu and T. M. Vu, Periodic solutions to NavierStokes equations on non-compact Einstein manifolds with negative curvature, Anal. Math. Phys. 11 (2021), no. 2, Paper No. 60, 17 pp.

[28] V. Pierfelice, The incompressible Navier-Stokes equations on non-compact manifolds, J. Geom. Anal. 27 (2017), no. 1, 577-617.

[29] G. Prodi, Qualche risultato riguardo alle equazioni di Navier-Stokes nel caso bidimensionale, Rend. Sem. Mat. Univ. Padova 30 (1960), 1-15.

[30] G. Prouse, Soluzioni periodiche dell'equazione di Navier-Stokes, Atti Accad. Naz. Lincei Rend. Cl. Sci. Fis. Mat. Nat. (8) 35 (1963), 443-447.

[31] J. Serrin, A note on the existence of periodic solutions of the Navier-Stokes equations, Arch. Rational Mech. Anal. 3 (1959), 120-122.

[32] Y. Taniuchi, On stability of periodic solutions of the Navier-Stokes equations in unbounded domains, Hokkaido Math. J. 28 (1999), no. 1, 147-173.

[33] - On the uniqueness of time-periodic solutions to the Navier-Stokes equations in unbounded domains, Math. Z. 261 (2009), no. 3, 597-615.

[34] M. E. Taylor, Partial Differential Equations III: Nonlinear equations, Second edition, Applied Mathematical Sciences 117, Springer, New York, 2011.

[35] G. Van Baalen and P. Wittwer, Time periodic solutions of the Navier-Stokes equations with nonzero constant boundary conditions at infinity, SIAM J. Math. Anal. 43 (2011), no. 4, 1787-1809.

[36] M. Yamazaki, The Navier-Stokes equations in the weak- $L^{n}$ space with time-dependent external force, Math. Ann. 317 (2000), no. 4, 635-675.

[37] V. I. Yudovich, Periodic motions of a viscous incompressible fluid, Soviet Math. Dokl. 1 (1960), 168-172.

[38] Q. S. Zhang, The ill-posed Navier-Stokes equation on connected sums of $\mathbb{R}^{3}$, Complex Var. Elliptic Equ. 51 (2006), no. 8-11, 1059-1063. 
Thieu Huy Nguyen

School of Applied Mathematics and Informatics, Hanoi University of Science and

Technology, Vien Toan ung dung va Tin hoc, Dai hoc Bach khoa Hanoi, 1 Dai Co Viet, Hanoi, Vietnam

E-mail address: huy.nguyenthieu@hust.edu.vn

Truong Xuan Pham and Thi Van Nguyen

Faculty of Information Technology, Department of Mathematics, Thuyloi University,

Khoa Cong nghe Thong tin, Bo mon Toan, Dai hoc Thuy loi, 175 Tay Son, Dong Da, Ha Noi, Vietnam

E-mail addresses: xuanpt@tlu.edu.vn, van@tlu.edu.vn

Thi Ngoc Ha Vu

School of Applied Mathematics and Informatics, Hanoi University of Science and Technology, Vien Toan ung dung va Tin hoc, Dai hoc Bach khoa Hanoi, 1 Dai Co Viet, Hanoi, Vietnam

E-mail address: ha.vuthingoc@hust.edu.vn 